Solar Physics

DOI: $10.1007 / \bullet \bullet \bullet \bullet \bullet-\bullet \bullet \bullet-\bullet \bullet \bullet-\bullet \bullet \bullet \bullet-\bullet$

\title{
Photospheric Magnetic Evolution in the WHI Active Regions
}

\author{
B.T. Welsch ${ }^{1} \cdot$ S. Christe ${ }^{2}$. J.M. McTiernan ${ }^{1}$
}

(C) Springer $\bullet \bullet \bullet \bullet$

\begin{abstract}
Sequences of line-of-sight (LOS) magnetograms recorded by the MichelsonDoppler Imager are used to quantitatively characterize photospheric magnetic structure and evolution in three active regions that rotated across the Sun's disk during the Whole Heliosphere Interval (WHI), in an attempt to relate the photospheric magnetic properties of these active regions to flares and coronal mass ejections (CMEs). Several approaches are used in our analysis, on scales ranging from whole active regions, to magnetic features, to supergranular scales, and, finally, to individual pixels. We calculated several parameterizations of magnetic structure and evolution that have previously been associated with flare and CME activity, including total unsigned magnetic flux, magnetic flux near polarity inversion lines, amount of cancelled flux, the "proxy Poynting flux," and helicity flux. To catalog flare events, we used flare lists derived from both GOES and RHESSI observations. By most such measures, AR 10988 should have been the most flare- and CME-productive active region, and AR 10989 the least. Observations, however, were not consistent with this expectation: ARs 10988 and 10989 produced similar numbers of flares, and AR 10989 also produced a few CMEs. These results highlight present limitations of statistics-based flare and CME forecasting tools that rely upon line-of-sight photospheric magnetic data alone.
\end{abstract}

Keywords: Flares, Dynamics; Helicity, Magnetic; Magnetic fields, Corona

\section{Characterizing Photospheric Magnetic Evolution}

Evolution of magnetic fields in solar active regions (ARs) affects the heliosphere in many ways. In the low corona, sudden magnetic evolution in flares and coronal mass ejections (CMEs) - which arise on timescales of minutes to hours - can launch powerful disturbances into interplanetary space. More gradual magnetic

\footnotetext{
${ }^{1}$ Space Sciences Laboratory, University of California, 7 Gauss Way, Berkeley, CA 94720-7450, U.S.A.

email: welsch@ssl.berkeley.edu

${ }^{2}$ NASA/Goddard Space Flight Center, Greenbelt, MD 20771, U.S.A.
} 
evolution, such as the effective diffusion and transport of active region magnetic flux over the photosphere, also drives evolution in heliospheric structure (affecting, e.g., the structure of the streamer belt and the heliospheric current sheet), on typical timescales of weeks and months. Consequently, studying the evolution of active-region magnetic fields is crucial to understanding the structure and dynamics of the heliosphere, beyond understanding solar activity itself.

Quantifying evolution of magnetic fields in photospheric magnetograms, in particular, can provide insights into heliospheric evolution. Photospheric magnetograms reveal cross sections of the large-scale structure of active regions, which extend from the solar interior out into the corona. The coupling of the photospheric field to the coronal field implies that magnetic evolution at the photosphere will induce evolution in the coronal field. Note, however, that the coronal magnetic field can evolve independently of the photospheric field; for instance, MHD instabilities that arise in the coronal field might trigger flares or CMEs (e.g. Forbes, 2000).

Here, we present analyses of several aspects of photospheric magnetic evolution in the three active regions (NOAA ARs 10987, 10988, and 10989) that crossed the solar disk during the Whole Heliosphere Interval (WHI), as observed in line-of-sight (LOS) magnetograms recorded with the Michelson-Doppler Imager (MDI) instrument (Scherrer et al., 1995) on the SOHO satellite. The data are described in Section 2, our analysis methods and results are discussed in Section 3, and we conclude by discussing the implications of these results in Section 4.

\section{Data}

\subsection{Magnetograms}

Our analysis of photospheric magnetic evolution in the WHI ARs began with selection of magnetograms from the database of full-disk, line-of-sight, 96-minutecadence, Level 1.8.2 magnetograms from MDI (http://soi.stanford.edu/magnetic/ Lev1.8/). These magnetograms are formed either from a single measurement, nominally of 30 seconds, or by summing measurements over five minutes. The 30 -second magnetograms are much more common in the dataset than the fiveminute magnetograms; and the former are noisier. To estimate the noise levels in each, we fit Gaussians to histograms of field strengths (which are actually pixel-averaged flux densities) in one sample magnetogram of each type, and took the noise level as $1 \sigma_{\text {fit }}$. Each sample magnetogram was smoothed with a $2 \mathrm{D}$, three-pixel boxcar prior to histogramming into $3 \mathrm{G}$ bins. While we expect noise to dominate the weakest-field pixels, and would therefore prefer to fit only the "core" of each histogram (Hagenaar et al., 1999), in practice using ranges smaller than $\pm 30 \mathrm{G}$ resulted in poor fits. Results fitting this range of field strengths differed little from those derived by fitting the whole histogram. Wholehistogram $\sigma_{\text {fit }}$ values were $\simeq 14 \mathrm{G}$ and $\simeq 8 \mathrm{G}$ for single and five-measurement magnetograms, respectively; the sample histograms used are plotted in Figure 1. Given the scarcity of the five-minute magnetograms, we assume a single noise level of $14 \mathrm{G}$ for simplicity. 


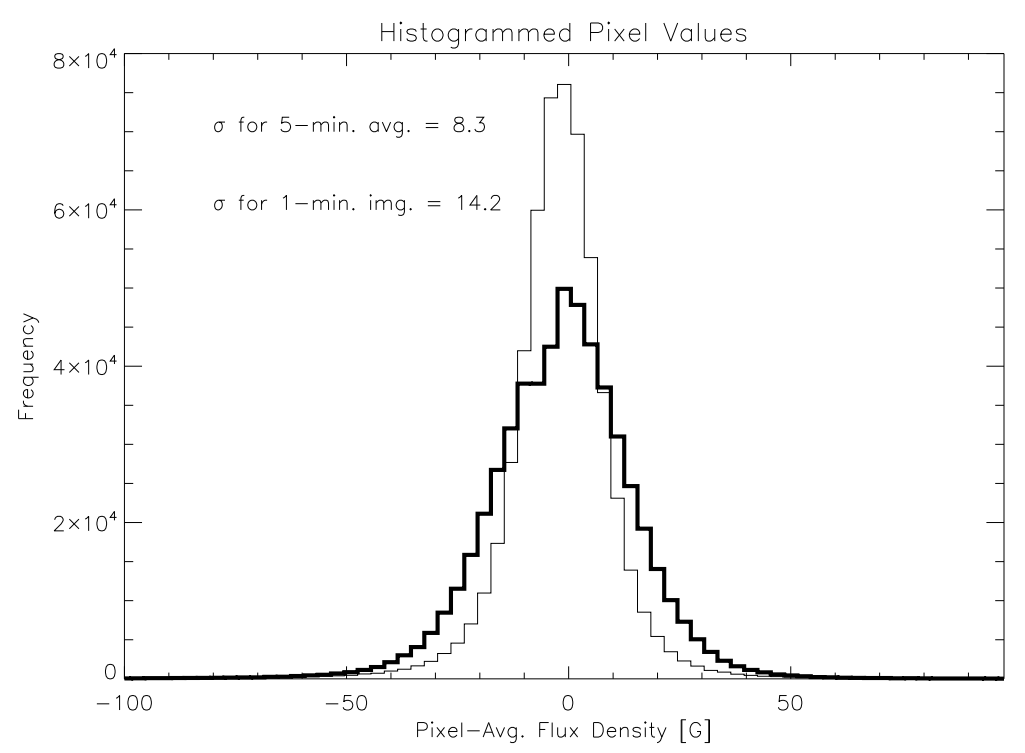

Figure 1. Histograms of pixel-average field strengths in single- (thick) and five-measurement (thin) magnetograms. Gaussian fits to the histograms can be used to estimate the noise levels in each, which are $\sim 14 \mathrm{G}$ and $\sim 8 \mathrm{G}$, respectively,

The MDI instrument measures the LOS field strength $\left[B_{\mathrm{LOS}}\right]$ averaged over each pixel. Tracking the evolution of the radial magnetic field $\left[B_{R}\right]$ requires estimating $B_{R}$, since only $B_{\mathrm{LOS}}$ was observed. We therefore assumed the magnetic field was radial, and applied cosine corrections to the LOS field in each pixel, $B_{R}=B_{\mathrm{LOS}} / \cos (\gamma)$, where $\gamma$ is the heliocentric angle from disk center to each pixel. To compensate for foreshortening, triangulation was used to interpolate the $B_{R}$ data - regularly gridded in the plane of the sky, but irregularly gridded in spherical coordinates $(\theta, \phi)$ on the solar surface - onto points $(x, y)$ corresponding to a regularly gridded Mercator projection of the spherical surface, following Welsch et al. (2009). This projection is conformal (and so locally preserves shape), which is necessary to ensure displacements measured by our tracking methods were not biased in direction. This reprojection distorts length scales, such that apparent displacements are exaggerated by a factor of the secant of the apparent latitude, which we corrected after tracking.

Since the MDI magnetograph only measures the LOS component of the photospheric magnetic field, we chose to analyze only magnetograms in which the target AR was within about $45^{\circ}$ of disk center. The first AR visible on the disk during WHI was AR 10987 on 24 March 2008, and we started our tracking analyses with the magnetogram recorded at 14:23 UT. Shortly thereafter, AR 10988 rotated onto the disk, followed by AR 10989. By 2 April, AR 10989 had no sunspots, but we analyzed magnetograms through 4 April, when AR 10989 was nearly $45^{\circ}$ from disk center, ending at 14:23 UT. The time ranges over which each AR was tracked are listed in Table 1. The top-most magnetogram in Figure 2 shows all three WHI ARs on the disk. The magnetograms in each of the 
Table 1. Intervals over which photospheric magnetic fields of WHI ARs were tracked on the central disk.

\begin{tabular}{ccc}
\hline NOAA AR & Tracking Start & Tracking End \\
\hline 10987 & 2008 Mar 24, 14:23UT & 2008 Mar 30, 04:47UT \\
10988 & 2008 Mar 26, 12:48UT & 2008 Apr 01, 06:23UT \\
10989 & 2008 Mar 28, 22:23UT & 2008 Apr 03, 14:23UT \\
\hline
\end{tabular}

subsequent rows of the same Figure show each WHI AR roughly one day after we began tracking it (left) and one day before we stopped tracking it (right).

\subsection{Flare and CME Activity}

Our primary goal is to relate properties of magnetic structure and evolution at the photosphere to energy release in the corona, in the form of flares and CMEs.

Webb et al. (2011) used several sources of data to compile a comprehensive list of CMEs over the WHI, and to determine their source regions. We used their results to determine CME productivity of the WHI ARs during the time intervals shown in Table 3. Only AR 10989 was CME-productive while tracked; it produced two during its tracking interval (and a further two in the hours just outside the interval over which it was tracked).

While CMEs are generally responsible for the strongest heliospheric disturbances (Gosling, 1993), flares can also affect the heliosphere. As detailed below, each WHI AR only produced relatively small flares during the interval over which it was tracked. But even weak flares can affect the heliosphere: for instance, small hard X-ray (HXR) bursts are often associated with interplanetary Type III radio bursts (e.g. Christe, Krucker, and Lin, 2008a), which arise from electrons escaping into the heliosphere (where they have been observed in situ).

To characterize flare activity in the WHI ARs, we first consulted records of soft X-ray (SXR) flare emission observed with the GOES satellites in the flare catalog maintained by NOAA (ftp://ftp.ngdc.noaa.gov/STP/SOLAR\_DATA/SOLAR_FLARES/ FLARES_XRAY/), and found that most of the relatively weak flares during WHI were not attributed to any source region, meaning this catalog did not accurately characterize each AR's flare activity. Next, we consulted Sam Freeland's Latest Events Archive (LEA: http://www.Imsal.com/solarsoft/latest_events_archive. html) which differences EUV images to determine flare-source locations. The LEA flare list identified the source ARs for many events that lacked source ARs in the GOES catalog. In the three cases where the NOAA and LEA source attributions disagreed, manual inspection of LEA difference images suggested the LEA attributions were probably correct. Some flares in the LEA list were incorrectly attributed to either: i) remnant ARs from previous rotations that were due to rotate back to the flares' positions when they occurred, or ii) ARs farther West than the true source AR. Both errors might arise from using active region locations from Solar Region Summaries (SRSs), jointly prepared once per day by NOAA's Space Weather Prediction Center (SWPC; http://www. 


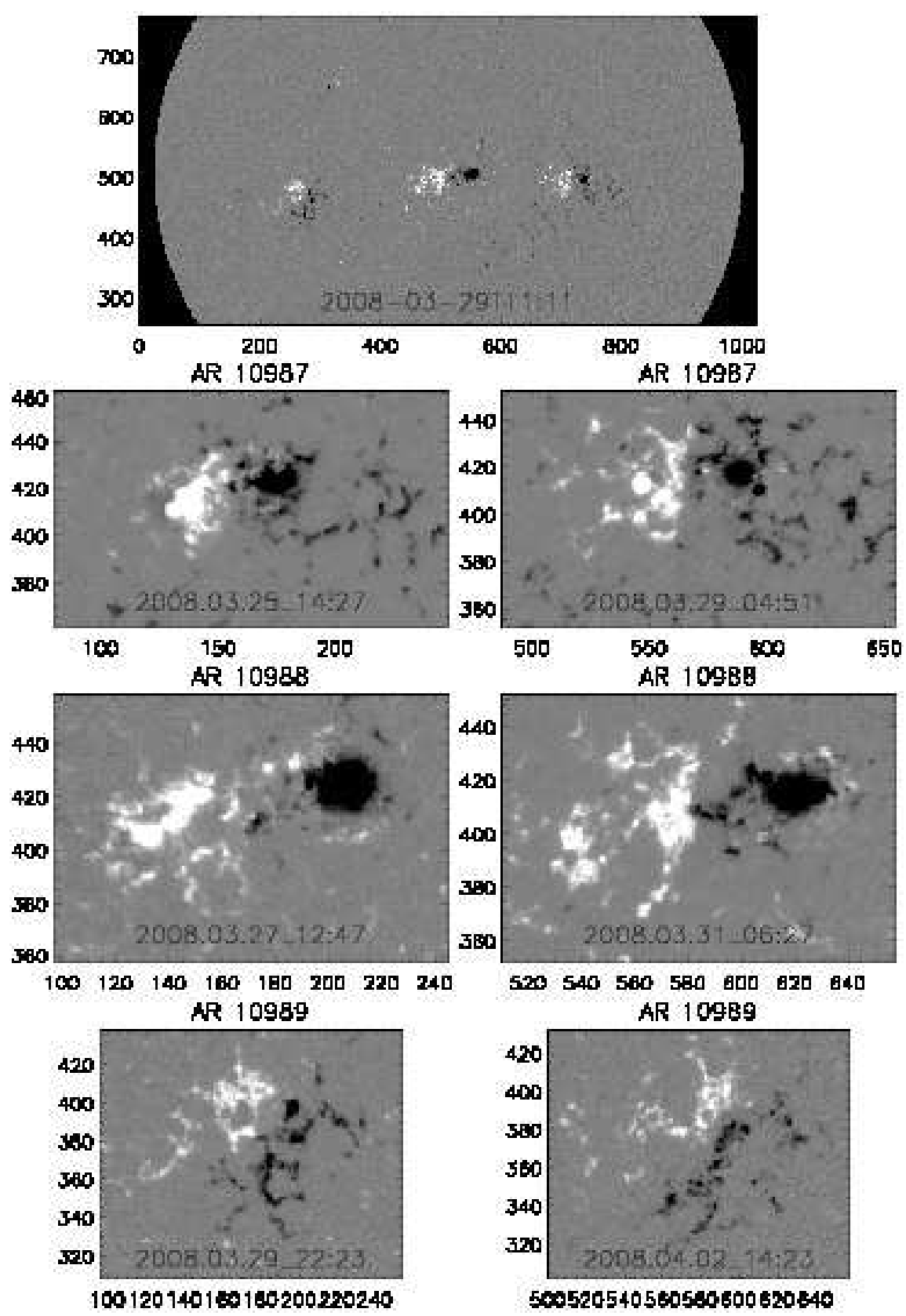

Figure 2. Top: This full-disk magnetogram, cropped at $\pm 30^{\circ}$, shows all three WHI ARs (from right to left, 10987, 10988, 10989). The cropped magnetograms in each of the subsequent rows show each AR roughly one day after we began tracking it (left) and one day before we stopped tracking it (right). Axes' labels are pixels from MDI full-disk magnetograms. 
swpc.noaa.gov/ftpdir/forecasts/SRS/README) and the U.S. Air Force, that were issued many hours before flare time. While source latitudes and longitudes in the LEA list appear accurate for the events we studied, AR source attributions from both the NOAA and LEA event lists contain errors.

A more robust approach to automatic identification of flares' source active regions uses data from the RHESSI satellite (Lin et al., 2002), which is capable of localizing the source regions of hard X-rays (HXRs) emitted in flares (as well as imaging HXR emission in high-fluence flares). Recent improvements in the algorithms used to identify microflares (short bursts of HXR emission in RHESSI's 6-12 keV energy band; see, e.g., Christe et al., 2008b), which are invariably associated with active regions, has enabled their inclusion in the most recent RHESSI flare catalog (http://hessi.ssl.berkeley.edu/hessidata/dbase/hessiflare_list.txt). To find the source AR for each flare in the RHESSI catalog, disk positions for the WHI ARs from the daily SRSs were used to develop linear models of for each AR's apparent latitude and longitude as a function of time. This approach enabled extrapolating the positions of ARs both before they were visible near the east limb and recognized as new ARs, and after they passed beyond the west limb. Flares were automatically associated by proximity to the nearest predicted AR location. Visual inspection of each automatic association confirmed that this method correctly assigned above-the-limb flares to ARs which, though not visible, were at or near the limb. Lightcurves from GOES were then searched to identify the transient X-ray enhancement associated with each RHESSI flare, and the enhancement's peak intensity was recorded.

Flares and source AR attributions are listed in Table 2, and plotted in Figure 3 , which shows the position of each RHESSI flare with respect to the solar disk, and its AR association. We note that most of the flares for AR 10988 occurred on the eastern disk, and many of the flares for 10989 occured above the east limb. Consequently, the LOS magnetograms from MDI cannot tell us much about the photospheric magnetic structure of these ARs during their most flare-productive intervals.

In Table 3, we summarize flare activity for each WHI AR separately for two time intervals: first, over the whole time range from appearance over the east limb to its disappearance around the west limb; and second, during the limited time interval that the AR was tracked. Not counting RHESSI flares for which a peak in the GOES lightcurve could not be automatically isolated, we found that ARs 10987, 10988, and 10999 produced 30, 44, and 28 flares, respectively, as they crossed the disk, and 9, 11, and 5 flares, respectively, while they were tracked across the central disk. Abramenko (2005) used a weighted, time-average of each AR's flare X-ray fluxes observed by GOES as a "flare index" to quantify AR flare activity; consistent with the definition of GOES flare classes, a power of 10 difference in weighting was used between flare classes. Given such a weighting, the M- and C-class flares produced by AR 10989 imply that its average flare flux greatly exceeds that of the other two ARs during either of the intervals in Table 3. 
Table 2. RHESSI HXR bursts in the $612 \mathrm{keV}$ channel during the WHI interval from the RHESSI flare list. Only those events with valid positions are shown in this list. Positions are listed in heliographic coordinates (in degrees) if on the disk or in heliocentric coordinates (in arcseconds) if above the limb. The associated GOES class is also shown for each event; some events occurred during GOES data gaps.

\begin{tabular}{|c|c|c|c|c|c|c|c|}
\hline$\#$ & Date & Start & Stop & Peak & Coordinates & GOES Class & Source AR \\
\hline 1 & 2008 Mar 23 & $18: 51$ & $18: 51$ & $18: 51$ & S08E79 & A 7.9 & 10988 \\
\hline 2 & 2008 Mar 23 & $23: 38$ & $23: 41$ & $23: 39$ & S08E50 & A6.5 & 10987 \\
\hline 3 & 2008 Mar 23 & $23: 44$ & $23: 48$ & $23: 45$ & S08E51 & B1.4 & 10987 \\
\hline 4 & 2008 Mar 23 & $23: 51$ & $23: 51$ & $23: 51$ & S07E50 & A6.4 & 10987 \\
\hline 5 & 2008 Mar 24 & $00: 24$ & $00: 25$ & $00: 25$ & S08E50 & B1.0 & 10987 \\
\hline 6 & 2008 Mar 24 & $01: 14$ & $01: 16$ & $01: 15$ & S07E48 & B1.0 & 10987 \\
\hline 7 & 2008 Mar 24 & $01: 18$ & $01: 22$ & 01:19 & S07E49 & B1.8 & 10987 \\
\hline 8 & 2008 Mar 24 & $01: 26$ & $01: 29$ & $01: 27$ & S08E50 & B1.3 & 10987 \\
\hline 9 & 2008 Mar 24 & $01: 32$ & $01: 33$ & 01:32 & S08E50 & B1.1 & 10987 \\
\hline 10 & 2008 Mar 24 & $01: 47$ & $01: 50$ & $01: 48$ & S08E76 & A9.3 & 10988 \\
\hline 11 & 2008 Mar 24 & $02: 46$ & $02: 53$ & $02: 47$ & S08E49 & B4.6 & 10987 \\
\hline 12 & 2008 Mar 24 & $02: 53$ & $02: 59$ & $02: 54$ & S08E48 & B2.8 & 10987 \\
\hline 13 & 2008 Mar 24 & $02: 59$ & 03:11 & 03:03 & S07E47 & B4.4 & 10987 \\
\hline 14 & 2008 Mar 24 & $06: 45$ & $06: 49$ & $06: 48$ & S07E44 & A 8.9 & 10987 \\
\hline 15 & 2008 Mar 24 & $07: 59$ & $08: 10$ & 08:04 & S07E45 & B2.3 & 10987 \\
\hline 16 & 2008 Mar 24 & 08:10 & $08: 36$ & $08: 16$ & S07E46 & B3.3 & 10987 \\
\hline 17 & 2008 Mar 24 & 10:08 & $10: 10$ & 10:09 & S07E43 & A 7.0 & 10987 \\
\hline 18 & 2008 Mar 24 & $11: 36$ & $11: 39$ & $11: 37$ & S08E71 & A 7.7 & 10988 \\
\hline 19 & 2008 Mar 24 & $11: 39$ & $11: 44$ & $11: 39$ & S08E68 & A6.4 & 10988 \\
\hline 20 & 2008 Mar 24 & $12: 56$ & $12: 57$ & $12: 56$ & S07E42 & A7.1 & 10987 \\
\hline 21 & 2008 Mar 24 & $13: 05$ & $13: 06$ & $13: 06$ & S08E67 & A 8.5 & 10988 \\
\hline 22 & 2008 Mar 24 & $14: 04$ & 14:07 & 14:06 & S08E67 & B1.1 & 10988 \\
\hline 23 & 2008 Mar 24 & $14: 47$ & $14: 49$ & $14: 48$ & S09E69 & A 7.2 & 10988 \\
\hline 24 & 2008 Mar 24 & $14: 53$ & $14: 58$ & $14: 55$ & S09E68 & A 7.9 & 10988 \\
\hline 25 & 2008 Mar 24 & $15: 40$ & $15: 41$ & $15: 40$ & S08E66 & A 7.2 & 10988 \\
\hline 26 & 2008 Mar 24 & $15: 56$ & $15: 58$ & $15: 57$ & S08E67 & B1.0 & 10988 \\
\hline 27 & 2008 Mar 24 & $16: 14$ & $16: 21$ & $16: 16$ & S07E40 & B1.2 & 10987 \\
\hline 28 & 2008 Mar 24 & $17: 13$ & $17: 14$ & $17: 13$ & S08E65 & A9.5 & 10988 \\
\hline 29 & 2008 Mar 24 & $17: 34$ & $17: 38$ & $17: 34$ & S08E65 & A9.4 & 10988 \\
\hline 30 & 2008 Mar 24 & 19:08 & $19: 13$ & 19:09 & $-964,-197$ & B1.0 & 10989 \\
\hline 31 & 2008 Mar 24 & $20: 52$ & $20: 54$ & $20: 53$ & S07E37 & B1.3 & 10987 \\
\hline 32 & 2008 Mar 24 & $21: 58$ & $22: 04$ & $21: 59$ & S07E63 & B1.7 & 10988 \\
\hline 33 & 2008 Mar 25 & $01: 59$ & 02:02 & 02:00 & S07E59 & B1.4 & 10988 \\
\hline 34 & 2008 Mar 25 & $04: 27$ & $04: 30$ & $04: 28$ & S07E59 & no data & 10988 \\
\hline 35 & 2008 Mar 25 & $04: 30$ & $04: 31$ & $04: 31$ & S07E59 & no data & 10988 \\
\hline 36 & 2008 Mar 25 & 04:50 & 05:07 & $04: 54$ & $-966,-182$ & B5.2 & 10989 \\
\hline 37 & 2008 Mar 25 & $07: 53$ & 08:04 & 08:01 & $-943,-207$ & B1.8 & 10989 \\
\hline 38 & 2008 Mar 25 & 09:31 & 09:35 & 09:34 & $-949,-172$ & B1.1 & 10989 \\
\hline 39 & 2008 Mar 25 & $11: 18$ & $11: 28$ & $11: 20$ & $-958,-139$ & B1.7 & 10989 \\
\hline 40 & 2008 Mar 25 & $13: 18$ & $13: 22$ & $13: 21$ & S08E53 & B1.2 & 10988 \\
\hline 41 & 2008 Mar 25 & $14: 44$ & $14: 47$ & $14: 45$ & S06E47 & B1.4 & 10988 \\
\hline 42 & 2008 Mar 25 & $14: 49$ & $14: 54$ & $14: 52$ & S11E84 & B2.3 & 10989 \\
\hline 43 & 2008 Mar 25 & $16: 10$ & $16: 12$ & $16: 10$ & S09E82 & B1.1 & 10989 \\
\hline 44 & 2008 Mar 25 & $16: 12$ & $16: 19$ & $16: 15$ & S08E48 & B1.1 & 10988 \\
\hline 45 & 2008 Mar 25 & $17: 36$ & $17: 41$ & $17: 38$ & S11E81 & B2.4 & 10989 \\
\hline 46 & 2008 Mar 25 & $18: 44$ & $19: 28$ & $18: 51$ & $-950,-178$ & M1.7 & 10989 \\
\hline 47 & 2008 Mar 25 & $19: 28$ & $19: 30$ & $19: 30$ & $-973,-189$ & C3.4 & 10989 \\
\hline
\end{tabular}


B.T. Welsch, J.M. McTiernan, and S.D. Christe

Table 2. Continued from previous page; see above for more information.

\begin{tabular}{|c|c|c|c|c|c|c|c|}
\hline \# & Date & Start & Stop & Peak & Coordinates & GOES Class & Source AR \\
\hline 48 & 2008 Mar 25 & $20: 20$ & $20: 28$ & $20: 21$ & $-974,-200$ & B9.1 & 10989 \\
\hline 49 & 2008 Mar 25 & 21:06 & $21: 08$ & $21: 08$ & $-982,-199$ & B4.9 & 10989 \\
\hline 50 & 2008 Mar 25 & $21: 56$ & $22: 12$ & $21: 57$ & $-987,-204$ & B3.4 & 10989 \\
\hline 51 & 2008 Mar 25 & $22: 12$ & $22: 27$ & $22: 18$ & $-989,-211$ & B3.1 & 10989 \\
\hline 52 & 2008 Mar 25 & $22: 27$ & $22: 43$ & $22: 29$ & $-990,-213$ & B2.8 & 10989 \\
\hline 53 & 2008 Mar 25 & $22: 43$ & $22: 47$ & $22: 47$ & $-985,-208$ & B2.5 & 10989 \\
\hline 54 & 2008 Mar 25 & $23: 32$ & $23: 42$ & $23: 35$ & $-992,-219$ & B2.7 & 10989 \\
\hline 55 & 2008 Mar 26 & 01:08 & 01:14 & 01:08 & $-100,-207$ & B1.5 & 10989 \\
\hline 56 & 2008 Mar 26 & $01: 21$ & $01: 23$ & $01: 22$ & $-963,-213$ & B1.4 & 10989 \\
\hline 57 & 2008 Mar 26 & 03:00 & 03:06 & 03:01 & S09E47 & B1.3 & 10988 \\
\hline 58 & 2008 Mar 26 & $03: 28$ & $03: 29$ & $03: 28$ & S07E18 & B1.3 & 10987 \\
\hline 59 & 2008 Mar 26 & $05: 19$ & $05: 20$ & $05: 20$ & S06E18 & B1.0 & 10987 \\
\hline 60 & 2008 Mar 26 & $06: 28$ & $06: 29$ & $06: 29$ & S07E43 & B1.1 & 10988 \\
\hline 61 & 2008 Mar 26 & $07: 59$ & 08:01 & $07: 59$ & S09E41 & A9.0 & 10988 \\
\hline 62 & 2008 Mar 26 & $11: 23$ & $11: 29$ & $11: 25$ & S08E40 & B1.9 & 10988 \\
\hline 63 & 2008 Mar 26 & $14: 21$ & $14: 34$ & $14: 21$ & S08E39 & B1.0 & 10988 \\
\hline 64 & 2008 Mar 26 & $17: 07$ & $17: 17$ & $17: 10$ & S07E36 & B1.2 & 10988 \\
\hline 65 & 2008 Mar 26 & $18: 53$ & 19:07 & $18: 55$ & S07E36 & B1.3 & 10988 \\
\hline 66 & 2008 Mar 26 & 19:07 & $19: 13$ & 19:09 & S06E35 & B1.2 & 10988 \\
\hline 67 & 2008 Mar 26 & $19: 17$ & $19: 21$ & $19: 19$ & S07E35 & B1.3 & 10988 \\
\hline 68 & 2008 Mar 27 & 01:35 & $01: 37$ & 01:35 & S11E33 & A 8.6 & 10988 \\
\hline 69 & 2008 Mar 27 & $02: 42$ & $02: 51$ & $02: 43$ & S06E30 & B1.5 & 10988 \\
\hline 70 & 2008 Mar 27 & $03: 36$ & $03: 37$ & $03: 36$ & S08E33 & no data & 10988 \\
\hline 71 & 2008 Mar 27 & $05: 17$ & $05: 19$ & $05: 18$ & S07E29 & A8.9 & 10988 \\
\hline 72 & 2008 Mar 27 & $11: 10$ & $11: 13$ & $11: 10$ & S06E26 & A 8.1 & 10988 \\
\hline 73 & 2008 Mar 27 & $15: 50$ & $15: 53$ & $15: 50$ & S05W03 & B1.3 & 10987 \\
\hline 74 & 2008 Mar 27 & $16: 19$ & $16: 20$ & $16: 19$ & S06W03 & B1.5 & 10987 \\
\hline 75 & 2008 Mar 28 & 01:11 & $01: 15$ & 01:11 & S05W07 & A6.5 & 10987 \\
\hline 76 & 2008 Mar 28 & 09:39 & 09:48 & 09:44 & S10W11 & B1.6 & 10987 \\
\hline 77 & 2008 Mar 28 & $10: 44$ & $10: 46$ & $10: 45$ & S07W12 & A 7.2 & 10987 \\
\hline 78 & 2008 Mar 28 & 14:01 & $14: 10$ & $14: 03$ & $\mathrm{~S} 14 \mathrm{E} 13$ & B1.2 & 10988 \\
\hline 79 & 2008 Mar 28 & $15: 27$ & $15: 28$ & $15: 28$ & S10E40 & A 5.8 & 10989 \\
\hline 80 & 2008 Mar 30 & $07: 55$ & $07: 56$ & $07: 55$ & S07W39 & A 5.6 & 10987 \\
\hline 81 & 2008 Mar 30 & $17: 32$ & $17: 36$ & $17: 36$ & S07W44 & B1.3 & 10987 \\
\hline 82 & 2008 Mar 30 & $20: 33$ & $20: 36$ & $20: 33$ & S07W48 & A 8.5 & 10987 \\
\hline 83 & 2008 Mar 30 & $23: 25$ & $23: 29$ & $23: 26$ & S07W49 & A 8.3 & 10987 \\
\hline 84 & 2008 Mar 31 & 06:05 & 06:06 & $06: 05$ & S07W54 & A 8.3 & 10987 \\
\hline 85 & 2008 Mar 31 & $06: 32$ & $06: 36$ & $06: 33$ & S08W23 & A 5.5 & 10988 \\
\hline 86 & 2008 Apr 01 & $00: 15$ & $00: 16$ & $00: 16$ & S11W05 & A 7.9 & 10989 \\
\hline 87 & 2008 Apr 01 & $20: 15$ & $20: 16$ & $20: 15$ & S11W15 & A6.5 & 10989 \\
\hline 88 & 2008 Apr 01 & $20: 18$ & $20: 22$ & $20: 20$ & S10W15 & B1.0 & 10989 \\
\hline 89 & 2008 Apr 02 & 03:09 & $03: 15$ & $03: 10$ & S09W49 & A 4.3 & 10988 \\
\hline 90 & 2008 Apr 02 & $12: 34$ & $12: 38$ & $12: 36$ & S09W55 & A 8.6 & 10988 \\
\hline 91 & 2008 Apr 02 & $15: 44$ & $15: 46$ & $15: 45$ & S08W56 & A 5.1 & 10988 \\
\hline 92 & 2008 Apr 02 & $16: 51$ & $16: 58$ & $16: 51$ & S08W61 & B1.0 & 10988 \\
\hline 93 & 2008 Apr 02 & 20:03 & $20: 13$ & 20:06 & S16W26 & B1.1 & 10989 \\
\hline 94 & 2008 Apr 02 & $22: 30$ & $22: 31$ & $22: 30$ & S09W61 & A6.5 & 10988 \\
\hline 95 & 2008 Apr 02 & $23: 40$ & $23: 53$ & $23: 43$ & S09W62 & B2.0 & 10988 \\
\hline 96 & 2008 Apr 03 & 01:12 & 01:50 & $01: 21$ & S11W32 & $\mathrm{C} 1.2$ & 10989 \\
\hline
\end{tabular}




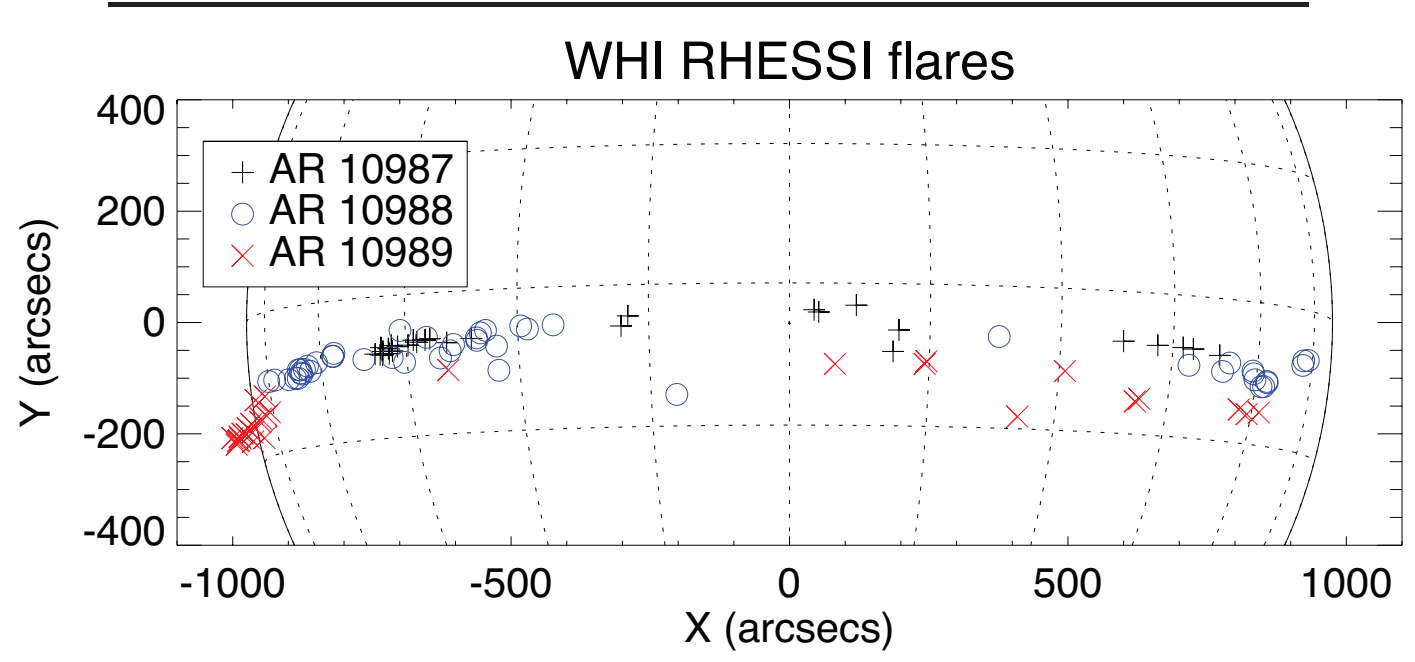

Figure 3. Positions and source ARs for flares identified by RHESSI.

Table 2. Continued from previous page; see above for more information.

\begin{tabular}{rlcccccc}
\hline \# & Date & Start & Stop & Peak & Coordinates & GOES Class & Source AR \\
\hline 97 & 2008 Apr 03 & $04: 56$ & $04: 57$ & $04: 57$ & S10W64 & A8.1 & 10988 \\
98 & 2008 Apr 03 & $05: 55$ & $06: 02$ & $05: 57$ & S09W65 & B1.2 & 10988 \\
99 & 2008 Apr 03 & $06: 02$ & $06: 10$ & $06: 07$ & S09W65 & A9.9 & 10988 \\
100 & 2008 Apr 03 & $07: 35$ & $07: 37$ & $07: 36$ & S10W63 & A4.2 & 10988 \\
101 & 2008 Apr 03 & $20: 00$ & $20: 11$ & $20: 04$ & S06W77 & no data & 10988 \\
102 & 2008 Apr 03 & $20: 15$ & $20: 20$ & $20: 17$ & S13W41 & no data & 10989 \\
103 & 2008 Apr 03 & $20: 20$ & $20: 28$ & $20: 23$ & S13W42 & no data & 10989 \\
104 & 2008 Apr 03 & $22: 09$ & $22: 14$ & $22: 11$ & S06W75 & A8.7 & 10988 \\
105 & 2008 Apr 03 & $22: 22$ & $22: 24$ & $22: 22$ & S06W75 & A5.8 & 10988 \\
106 & 2008 Apr 05 & $03: 13$ & $03: 17$ & $03: 13$ & S13W59 & A5.1 & 10989 \\
107 & 2008 Apr 05 & $05: 33$ & $05: 37$ & $05: 33$ & S13W61 & A6.4 & 10989 \\
108 & 2008 Apr 05 & $11: 02$ & $11: 16$ & $11: 05$ & S12W64 & B1.3 & 10989 \\
\hline
\end{tabular}

\section{Analysis of Magnetic Structure and Evolution}

We quantitatively analyzed magnetic evolution in these three ARs in several ways, which we present roughly in order of decreasing length scale: starting from whole-active-region measures of magnetic structure and evolution, we consider evolution on progressively smaller spatial scales, down to individual MDI pixels.

\subsection{Large Scale Structure and Evolution}

Barnes and Leka (2008), Leka and Barnes (2007), and Welsch et al. (2009) have noted that total unsigned radial magnetic flux $[\Phi]$ in an AR is strongly correlated with its flare productivity. 
Table 3. Observed Flares and CMEs from WHI ARs, from limb to limb (top three rows), and while tracked (bottom three rows). A, B, C, and M denote GOES flare classes. (RHESSI flares during GOES data gaps are not included in these tallies.) CMEs within a few hours before or after the start or end times shown are included in parentheses before or after the frequency during the interval, respectively. The M- and C-class flares produced by AR 10989 imply that its flare index, a measure of AR flare productivity (Abramenko, 2005), exceeds those of ARs 10987 and 10988.

\begin{tabular}{lllllllcc}
\hline AR & Start Obs. & End Obs. & A & B & C & M & Tot. Flares & CMEs \\
\hline 10987 & Mar 23, 23:39UT & Apr 05, 11:17UT & 11 & 19 & & 30 & $0(1)$ \\
10988 & Mar 23, 18:51UT & Apr 05, 11:17UT & 24 & 20 & & & 44 & $(1) 4$ \\
10989 & Mar 24, 19:09UT & Apr 05, 11:17UT & 5 & 20 & 2 & 1 & 28 & $8(1)$ \\
\hline \multirow{2}{*}{10987} & Mar 24, 14:23UT & Mar 30, 04:47UT & 2 & 7 & & 9 & 0 \\
10988 & Mar 26, 12:48UT & Apr 01, 06:23UT & 4 & 7 & & & 11 & 0 \\
10989 & Mar 28, 22:23UT & Apr 03, 14:23UT & 2 & 2 & 1 & & 5 & $(1) 2(1)$ \\
\hline
\end{tabular}

We calculated the total unsigned estimated radial flux, $\left[\Phi=\int \mathrm{d} A\left|B_{R}\right|\right]$, in each of the WHI ARs over the time interval each was tracked, compensating for distortion in pixel areas due to the Mercator projection. These unsigned fluxes are plotted in Figure 4. The average values of unsigned flux for ARs 10987, 10988, and 10989 were 1.6, 2.0, and $1.2 \times 10^{22} \mathrm{Mx}$, respectively, over the interval when each was tracked. To minimize the effects of noise, only pixels with unsigned field strength greater than $40 \mathrm{G}$ were included in the sums; this is approximately three times our assumed noise level of 14G. Assuming the uncorrelated, uniform per-pixel noise level on flux of $\sigma_{0}=14 \mathrm{G}(\Delta x)^{2}$, where $\Delta x$ is the width of MDI full-disk pixels, a formal estimate of the error in total flux would be $\sigma_{\text {sum }}=\sqrt{N_{>40 G}} \sigma_{0}$; this is typically $<10^{20} \mathrm{Mx}$, which is not visible on these plots. Dips in the plots arise from lower noise levels in magnetograms averaged over five measurements, and give a better estimate of the noise level. Also, the magnetic field is highly correlated from frame to frame at 96 minute cadences (Welsch et al., 2009), implying frame-to-frame variations in total flux also indicate noise levels. Note that MDI has a known problem with saturation in strong-field regions (Liu, Norton, and Scherrer, 2007), such that flux values reported here might be lower than the actual flux (Wang et al., 2009).

Based upon total unsigned flux alone, AR 10988 should have been the most active, and AR 10989 the least.

Schrijver (2007) argued that strong magnetic fields concentrated near polarity inversion lines (PILs) of the photospheric LOS magnetic field are closely associated with the occurrence of large flares. He developed a method to quantify the total unsigned magnetic flux $[R]$ near strong-field polarity inversion lines (SPILs) of ARs, by generating a weighting map of proximity to SPILs and summing the product of the weighting map and unsigned LOS magnetic flux. Because strong fields along LOS PILs are correlated with strong gradients in LOS fields across PILs (Falconer, Moore, and Gary, 2003), the $R$ parameter is quantitatively related to the length of strong-gradient PILs, which has been found by Falconer, Moore, and Gary (2003) and Falconer, Moore, and Gary (2006) to be associated 


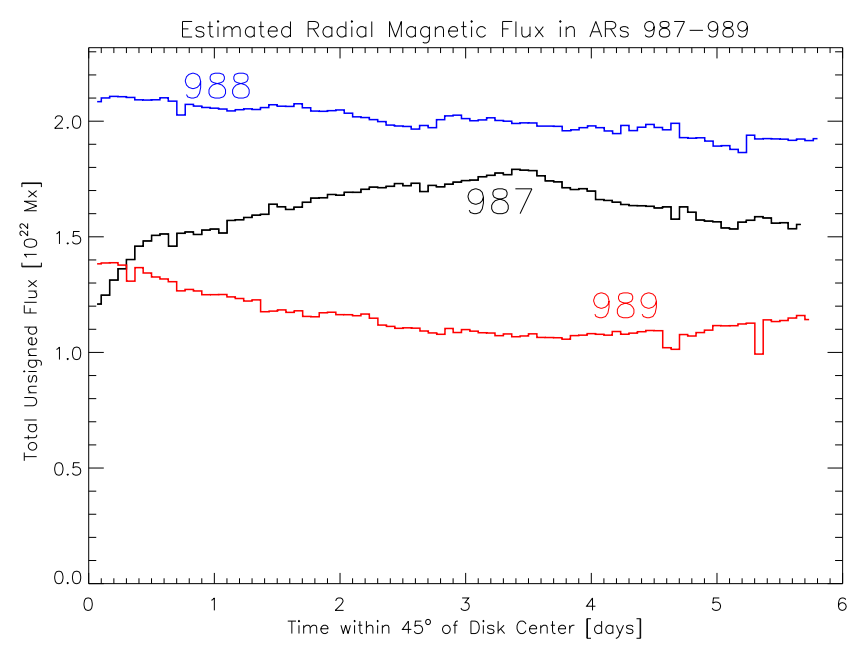

Figure 4. Total unsigned flux $[\Phi]$ versus time in the three WHI ARs, as each crossed the central solar disk. Dips arise from lower noise levels in magnetograms averaged over five measurements. Larger values of $\Phi$ have been associated with greater flare activity.

with CMEs. Barnes and Leka (2008) and Welsch et al. (2009) also found $R$ to be associated with flare activity.

Emulating Schrijver's approach here, we computed $R$ values for each of the WHI ARs as they crossed the disk, choosing (as he did) a strong-field threshold of $150 \mathrm{G}$ and FWHM of $15 \mathrm{Mm}$ in the Gaussian used in the convolution to compute the SPIL weighting map (see Welsch and Li, 2008 for more details of the procedure). Our results are plotted in Figure 5. Note that our values of $R$ are in units of Mx; Schrijver's were in units of G, summed over the weighting map. The MDI disk-center pixel area of $\simeq 2.2 \times 10^{16} \mathrm{~cm}^{2}$ is an approximate conversion factor. Note also that Schrijver (2007) apparently used MDI Level 1.8.0 magnetograms, while we use Level 1.8.2 magnetograms (see http://soi.stanford.edu/magnetic/Lev1.8/). Since absolute flux densities in the latter are higher than in the former by a spatially varying factor of approximately 1.6, our $R$ values cannot easily be compared with Schrijver's. As with estimating total unsigned flux, uncertainties in $R$ computed assuming an uncorrelated, uniform per-pixel noise level $\left[\sigma_{0}\right]$ are are too small to be visible on the plot. Instead, we have crudely estimated uncertainties from the $R$ values themselves by computing the standard error in the mean with a five-point boxcar window. This simplistic approach is reasonable because, as noted by Welsch et al.(2009), changes in magnetic fields are relatively minor over a few hours, so successive measurements of $R$ can be interpreted as repeated, independent measurements of nearly the same physical value.

As with total unsigned flux, expectations of flare activity based upon $R$ values would suggest AR 10988 should be most active, and AR 10989 should be least active. 


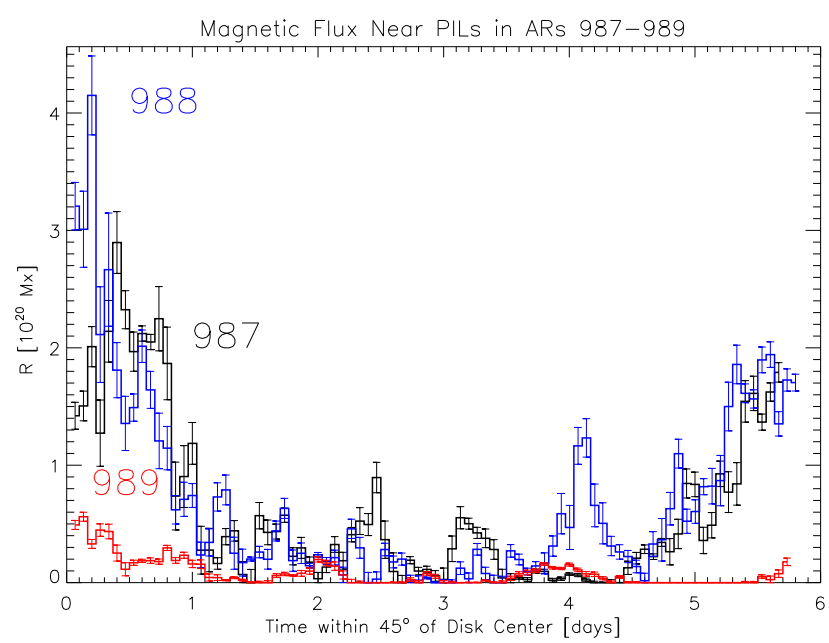

Figure 5. Total unsigned flux $[R]$ near strong-field polarity inversion lines (SPILs) versus time in the three WHI ARs, as each crossed the central solar disk. Large values of $R$ have been associated with flare activity.

\subsection{Intermediate-Scale Evolution: Feature Tracking}

Automated tracking of "features" in magnetogram sequences has been used to understand processes governing evolution of the photospheric magnetic field, including flux emergence and dispersal (e.g. DeForest et al., 2007 and references therein). Features in magnetograms have been identified in several ways, for instance by: "clumping" collections of contiguous, like-polarity, above-threshold pixels (e.g. Parnell et al., 2009); "downhill" segmentation, which identifies distinct "hilltops" in absolute field strength (e.g. Welsch and Longcope, 2003); and curvature, identifying the convex cores of hilltops in absolute field strength (e.g. Hagenaar et al., 1999). Tracking involves the association of identified features between successive frames.

Feature tracking can be used to identify episodes of flux cancellation, in which closely spaced, opposite-polarity features simultaneously appear to lose flux (Livi, Wang, and Martin, 1985). Since flux cancellation has been observed in prominence formation (Martin, 1998), shown to increase magnetic free energy in some circumstances (Welsch, 2006), and proposed as a CME initiation mechanism (e.g. Linker et al., 2003), we tracked downhill features in magnetogram sequences of the WHI ARs, to investigate cancellation rates in each AR.

Using the YAFTA feature-tracking code (Welsch and Longcope, 2003, DeForest et al., 2007; software and documentation are online at http://solarmuri. ssl.berkeley.edu/ welsch/public/software/YAFTA/) we only included pixels above $35 \mathrm{G}$ (a $2.5 \sigma$ threshold) in features, and required each feature to have at least four pixels, and at least one $70 \mathrm{G}$ pixel (a $5 \sigma$ peak threshold). We also required features to either persist for at least four frames, or interact with (fragment from, or merge with) a feature that persisted for four frames. The outlines of features identified in two successive magnetograms from AR 10987 are plotted over grayscale images 
of the magnetic field in Figure 6. The outlines are color coded by feature label, and features have been matched between these magnetograms. Different-color outlines imply distinct features were identified; same-color outlines generally imply matching features between frames, although the color palette used is limited, so spurious color matches are possible. While substantial evolution has occurred over the 96 minutes between these magnetograms, $90 \%$ of features in the first magnetogram were identified in the second, which is a typical survival rate.

Having tracked the three ARs' magnetogram sequences, we then attempted to quantify canceling features in each. We first found opposite-polarity features in close proximity by looping over positive features, applying IDL's dilate function, with a $(3 \times 3)$ structuring element, to each feature's pixels; and seeking instances of overlap between the dilated feature's pixels and pixels of negative features. This essentially searched for negative features in nearest-neighbor pixels on the periphery of each positive feature. Hence, our definition of close proximity for defining cancellation is MDI's pixel size, $\simeq 2^{\prime \prime} \simeq 1.4 \mathrm{Mm}$. For a pair of close features to have partially cancelled over a time step, we further required the features' centers of flux to approach, and the flux of both features to decrease - and by doing so, we have implicitly assumed that neither feature disappears completely over a cancellation step.

This approach is subject to many sources of error. Thresholds imposed on field strengths in features imply that some magnetic flux is not included in any feature. Features' positions jitter, and their fluxes fluctuate, both from noise and true evolution. Further, along the polarity-inversion lines where canceling features are typically identified, each feature often interacts with several neighboring like-polarity features, and possibly other opposite-polarity features, perhaps exchanging flux or canceling with those features, too. Hence, there are many essentially random sources of changes in features' fluxes and locations. With higher-cadence data, additional constraints might be imposed, e.g. meeting our cancellation criteria over several time steps. However, a more-detailed analysis of cancellation in higher-cadence data lies outside the purview of this study. Further, we are primarily interested in comparing cancelled flux between the WHI ARs, not establishing absolute amounts of flux cancelled.

Recognizing that our approach to quantifying cancelled flux is subject to large uncertainties, we computed estimates in three ways: $i$ ) first, for each pair of canceling features identified, we took the minimum flux loss from the pair as the cancelled flux in that event, and summed these fluxes from all cancellation events; ii) we limited our analysis to pairs of features that were observed to cancel over at least two steps, and took the average flux losses from such events; and iii) again restricting our attention to features that were observed to cancel over at least two steps, we summed the minimum flux lost from each pair in each event. The third approach is presumably the most restrictive method of estimating cancelled flux.

For each AR, our tabulation of the total number of cancellation events, the number of multi-step cancellation events, and estimates for cancelled flux are listed in Table 4. Variations between the estimates for a single AR provide a measure of the uncertainties, although as noted above, the method used for the 

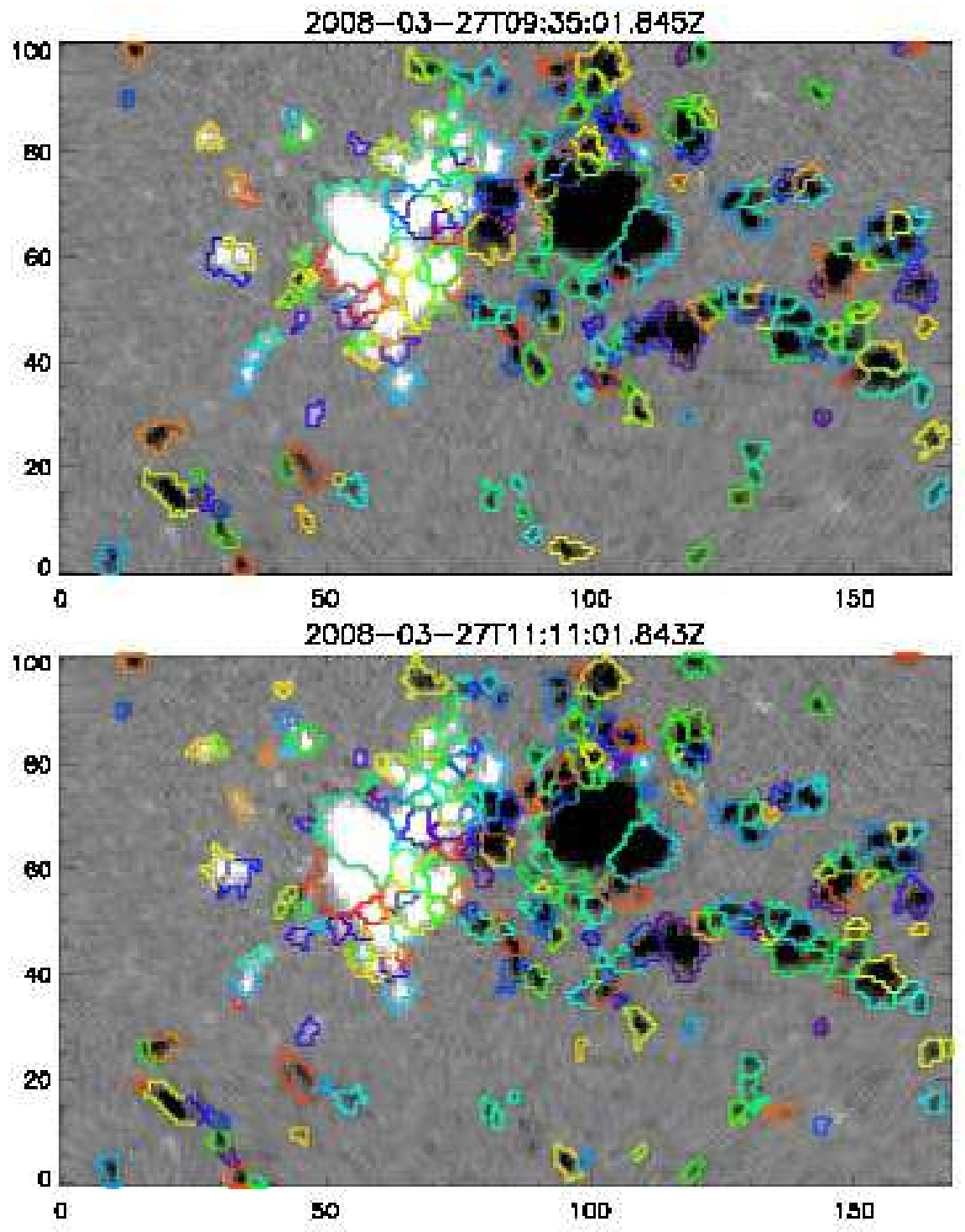

Figure 6. The outlines of features — "hills" in absolute field strength — identified in two successive magnetograms from AR 10987 are plotted over grayscale images of the magnetic field (white is positive polarity, black negative; saturation is set at $\pm 250 \mathrm{G}$ ). The outlines are color coded by feature label, and features have been matched between these magnetograms. Different-color outlines imply distinct features were identified; same-color outlines generally imply matching features between frames. 
Table 4. Estimates of flux cancelled in the WHI ARs, computed three ways (see text); cancelled flux values are in units of $\left[10^{20} \mathrm{Mx}\right]$.

\begin{tabular}{cccccc}
\hline AR & \# Events & \# Multi-step & $\begin{array}{c}\text { All events, } \\
\sum \min (\delta \Phi)\end{array}$ & $\begin{array}{c}\text { Multi-step, } \\
\sum \operatorname{avg}(\delta \Phi)\end{array}$ & $\begin{array}{c}\text { Multi-step, } \\
\sum \min (\delta \Phi)\end{array}$ \\
\hline 10987 & 58 & 10 & 5.0 & 4.0 & 1.9 \\
10988 & 110 & 21 & 8.0 & 8.1 & 3.4 \\
10989 & 42 & 5 & 2.9 & 1.6 & 0.8 \\
\hline
\end{tabular}

third estimate is the most restrictive. If our identification of canceling features were essentially random, and mean feature fluxes in all three ARs were the same, then our estimates of cancellation events and cancelled fluxes would follow the ratio of unsigned magnetic fluxes in the ARs, 1.4:1.7:1 (see Section 3.1 above). While the numbers in each column do follow the ordering of these ratios, there is an excess of both cancellation events and cancelled flux in AR 10988 beyond that expected from variations in AR flux alone.

As with both total unsigned flux and unsigned flux $[R]$ near SPILs, our analysis of flux cancellation in the WHI ARs suggests that AR 10988 should have been the most active, and AR 10989 the least.

\subsection{Pixel-Scale Evolution}

\subsubsection{Estimating Velocities}

According to Faraday's law, evolution of the radial magnetic field at the photosphere is governed by the curl of the electric field there,

$$
\partial_{t} B_{r}=-c(\nabla \times \mathbf{E})_{r}
$$

Assuming that the electric field is ideal (equivalently, that the plasma's conductivity is infinite) implies $\mathbf{E}=-(\mathbf{v} \times \mathbf{B}) / c$, so evolution of the radial magnetic field can be related to horizontal variations in the velocity and magnetic fields by the ideal induction equation,

$$
\begin{aligned}
\partial_{t} B_{r} & =[\nabla \times(\mathbf{v} \times \mathbf{B})]_{r} \\
& =-\nabla_{h} \cdot\left(\mathbf{v}_{h} B_{r}-v_{r} \mathbf{B}_{h}\right) .
\end{aligned}
$$

The radial flux $S_{r}$ of magnetic energy across the photosphere (the Poynting flux) depends upon $\mathbf{E}$, and in the ideal approximation $\mathbf{v}$,

$$
S_{r}=c[\mathbf{E} \times \mathbf{B}]_{r} / 4 \pi=\left[v_{r} B_{h}^{2}-\left(\mathbf{v}_{h} \cdot \mathbf{B}_{h}\right) B_{r}\right] / 4 \pi,
$$

as does the rate of change of relative magnetic helicity (Berger and Field, 1984) in the corona $\mathrm{d} H / \mathrm{d} t$ due to the helicity flux across the photosphere,

$$
\frac{\mathrm{d} H}{\mathrm{~d} t}=2 \int \mathrm{d} A\left[\left(\mathbf{A}_{h}^{P} \cdot \mathbf{B}_{h}\right) v_{r}-\left(\mathbf{A}_{h}^{P} \cdot \mathbf{v}_{h}\right) B_{r}\right],
$$


where: the integration runs over the photosphere in the region of non-zero $\mathbf{v}$ and $\mathbf{B} ; \mathbf{A}^{P}$ is the vector potential of the current-free (and therefore curl-free, or "potential") magnetic field $\mathbf{B}^{P}$ that matches the observed photospheric radial field $B_{r}$, i.e. $\nabla_{h} \times \mathbf{A}_{h}^{P}=B_{r}^{P}$; and $A_{r}^{P}=0=\nabla_{h} \cdot \mathbf{A}_{h}^{P}$.

Démoulin and Berger (2003) argued that the "footpoints" of magnetic fields anchored in the photosphere appear to move in magnetograms with an apparent footpoint velocity $\mathbf{u}$, which is related to the plasma velocity $\mathbf{v}$ by

$$
\mathbf{u} \equiv \mathbf{v}_{h}-\left(v_{r} / B_{r}\right) \mathbf{B}_{h} .
$$

They further argued that tracking methods applied to magnetograms, such as local correlation tracking (e.g. Chae, 2001), would estimate $\mathbf{u}$, not $\mathbf{v}_{h}$. Welsch (2006) referred to $\mathbf{u}$ as the flux transport velocity; $\mathbf{u} B_{r}$ has units of a flux transport rate (maxwells per unit length per unit time). Note that Equation (6) is a matter of definition, and so is made without approximation. In terms of $\mathbf{u}$, Equation (3) can be written in a form analogous to the continuity equation,

$$
\partial_{t} B_{r}+\nabla \cdot\left(\mathbf{u} B_{r}\right)=0
$$

The statement that tracking methods will accurately estimate $\mathbf{u}$, however, is a testable assertion; Schuck (2008) argued, in fact, that some "optical flow" methods are relatively insensitive to radial flows, so will return a biased estimate $\tilde{\mathbf{u}}$ of the true $\mathbf{u}$. Tests of several velocity estimation methods conducted by Welsch et al. (2007) using synthetic data from MHD simulations, in which the true velocities were known, demonstrated that such methods were far from perfect, but that their flow estimates were highly correlated with the true flows.

In terms of the flux transport velocity, the Poynting and helicity fluxes in Equations (4) and (5) become

$$
\begin{aligned}
S_{r} & =-\left(\mathbf{B}_{h} \cdot \mathbf{u}_{h}\right) B_{r} / 4 \pi \\
\frac{\mathrm{d} H}{\mathrm{~d} t} & =-2 \int \mathrm{d} A\left(\mathbf{A}_{h}^{P} \cdot \mathbf{u}_{h}\right) B_{r} .
\end{aligned}
$$

Note that estimating the Poynting flux requires knowledge of the horizontal magnetic field.

The flux transport velocity can be estimated from magnetogram sequences, with finite cadence $\Delta t$ and pixel size $\Delta x$, by applying a finite difference approximation to Equation (3),

$$
\frac{\Delta B_{z}}{\Delta t}+\frac{\Delta \cdot\left(\mathbf{u} B_{r}\right)}{\Delta x}=0
$$

where $\boldsymbol{\Delta}$ is the spatial finite-difference operator. If a typical flow speed is $v_{0}$, then the pixel-crossing time is $\tau_{0}=v_{0} \Delta x$. If $\Delta t<\tau_{0}$, then Equation (10) approximates Equation (7); if, however, $\Delta t>\tau_{0}$, the approximation can fail. This is analogous to the Courant-Friedrichs-Lewy (CFL) condition required for numerical stability in computational fluid dynamics.

Welsch et al. (2009) used Equation (10) as the basis for applying two tracking methods, Fourier local correlation tracking (FLCT: Welsch et al., 2004,Fisher 
and Welsch, 2008) and the differential affine velocity estimator (DAVE: Schuck, 2006), to sequences of 96-minute MDI full-disk magnetograms (similar to the current data, but Level 1.8.0 instead of Level 1.8.2). Both methods employed similar windowing parameters (eight and nine pixels for FLCT and DAVE, respectively) to localize the magnetic data used in estimating the flow at a given pixel, which probably has the effect of averaging over smaller-scale flows. Correlation coefficients between the field-weighted FLCT and DAVE flows' $x-$ and $y$-components were $>0.7$ over the set of all the flows computed by Welsch $e t$ al.(2009), implying consistency between flow estimates using distinct methods. Mean and median speeds for FLCT were $\simeq 65 \mathrm{~m} \mathrm{sec}^{-1}$, and for DAVE were $\simeq 105 \mathrm{~m} \mathrm{sec}^{-1}$; evidently, DAVE speeds are higher than those estimated with FLCT. Given the $\simeq 1.4 \mathrm{Mm}$ disk-center width of MDI full-disk pixels, these speeds imply pixel-crossing times of $\sim 4-6 \mathrm{hr}$. Welsch et al. (2009) also found that flow directions remained significantly correlated over similar time scales. Both of these facts imply that 96-minute cadence, full-disk MDI data can be used with Equation (10) as a valid approximation of Equation (7).

We applied FLCT to the 96-minute cadence MDI full-disk magnetograms within 23 Mar 2008, 00:00:00UT - 4 Apr 2008, 00:00:00UT from the WHI interval, with a windowing parameter of eight pixels, an absolute field-strength threshold for tracking of $50 \mathrm{G}$, and a low-pass spatial wavenumber roll-off parameter of 0.25 (see Fisher and Welsch, 2008). Pixels out to $45^{\circ}$ from disk center were tracked. This choice of windowing parameter corresponds to $\simeq 10 \mathrm{Mm}$, a scale much larger than granules, but slightly smaller than supergranules (Hagenaar, Schrijver, and Title, 1997).

Data cubes covering each of the WHI ARs' magnetic fields and their corresponding flows were then extracted from the full-disk magnetograms and innerdisk flow maps for the time intervals listed in the bottom three rows of Table 3. Figure 7 shows two successive FLCT flow maps from the central, strong-field portion of AR 10987, from the same magnetograms shown in Figure 6. Some evolution in the flow pattern can be seen over the 96 minutes between the flow maps; but flows in many regions appear qualitatively similar at both times.

\subsubsection{Proxy Poynting Flux}

Welsch et al. (2009) analyzed relationships of flare activity with photospheric magnetic field and flow properties in a sample of a few dozen ARs. Of several quantities that they investigated, they found Schrijver's $R$ value and a quantity proportional to $\sum\left(u B_{R}^{2}\right)$, where $u=|\mathbf{u}|$, and the sum runs over the AR magnetograms, to be most strongly associated with flare activity. Because $u B_{R}^{2}$ has the same dimensions as the Poynting flux of magnetic energy $\left(\mathrm{erg} \mathrm{cm}^{-2} \mathrm{sec}^{-1}\right)$, Welsch et al. (2009) called this quantity the proxy Poynting flux. For consistency with Equation (8), we define the proxy Poynting flux here to be $S_{R} \equiv\left(u B_{R}^{2}\right) / 4 \pi$. (Note that this definition differs from that of Welsch et al.(2009), who defined $S_{R}$ to include a sum over pixels - a total flux, instead of a flux density - and did not include $4 \pi$.) Li et al. (2010) estimated the proxy Poynting flux in AR 8038 around the time of the well-known 12 May 1997 flare/CME, and found the cumulative flux reached $\simeq 10^{32}$ erg over the four-day interval before the eruption. 

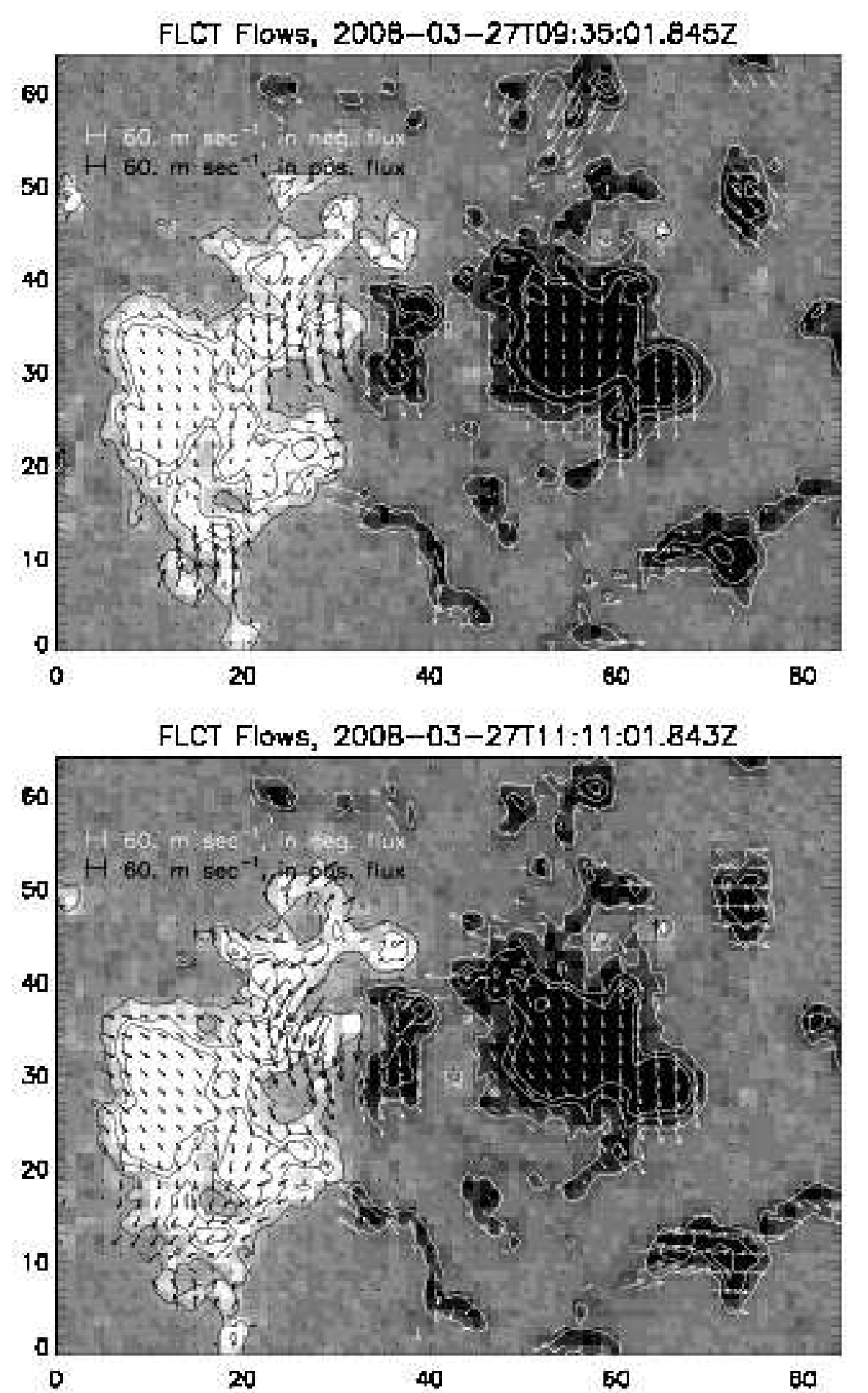

Figure 7. Two successive FLCT flow maps from the central, strong-field portion of AR 10987, from the same magnetograms shown in Figure 6. Background grayscale shows estimated radial field strength, white showing positive flux, black negative, with saturation set at $\pm 250 \mathrm{G}$. White/black contours correspond to $\pm 100, \pm 300$, and $\pm 500 \mathrm{G}$. For clarity, only every other flow vector is plotted. Some evolution in the flow pattern can be seen; but flows in many regions appear generally similar at both times. 
What is the physical basis for the relationship between the proxy Poynting flux and flare activity? Leaving aside the likelihood that estimates of $B_{r}$ and $\mathbf{u}$ are imperfect, in principle, $S_{R}$ corresponds to part of the horizontal Poynting flux $\left[S_{h}\right]$ (additional contributions arise from terms containing $\mathbf{B}_{h}$ ). The magnetic energy that powers flares and CMEs enters the corona from the solar interior, as an outward, radial Poynting flux $\left[S_{r}\right]$. It is plausible that there should be a correlation between $S_{r}$ and flare/CME activity, although the corona can store magnetic energy, so there might typically be a latency between the introduction of magnetic energy into the corona and its release in flares/CMEs. For instance, Longcope et al. (2005) and Schrijver et al. (2005) report latency times associated with active-region magnetic fields of $\sim 24$ hours. (And in quiet-Sun regions, filaments/ prominences, which are interpreted as current-carrying [non-potential] magnetic field structures, can persist in the corona for weeks.) It is also plausible that the radial Poynting flux $\left[S_{r}\right]$ is significantly correlated with $\left[S_{h}\right]$ and/or the proxy flux $\left[S_{R}\right]$, but no quantitative theoretical or observational characterization of such a correlation has been presented.

Using the FLCT flows we estimated for each WHI AR, we computed the proxy Poynting flux in that AR over the time interval between each tracked magnetogram pair. We then multiplied by (reprojection- corrected) pixel area $\Delta A$ and the time interval $\Delta t$ between magnetograms, and summed over all pixels to express our results in terms of ergs of energy transported. Results are plotted in the top panel of Figure 8; in the bottom panel, we plot the cumulative proxy Poynting fluxes over time. As with $R$, we estimated uncertainties in the top panel from a running-boxcar computation of the standard error in the mean. Assuming a combined fractional uncertainty of 0.6 for the terms in the product $u B_{R}^{2}$ in each pixel resulted in error bars smaller than those shown. Summing the uncertainties in the top panel in quadrature for the cumulative result shown in the bottom panel yields error bars about the size of the steps in the bottom panel.

Unlike the true Poynting flux, which can be positive or negative (i.e. radially outward or inward), the proxy Poynting flux is only positive. To the extent that the proxy Poynting flux reflects the outward radial flux of magnetic energy, the values in these plots suggest the order of magnitude of energy transport in the WHI ARs.

As with other magnetic parameters of the WHI ARs, values of the proxy Poynting flux suggest AR 10988 should have been the most active, and AR 10989 the least.

\subsubsection{Helicity Flux}

Magnetic helicity is approximately conserved even when magnetic reconnection occurs, meaning that it is not readily dissipated in the corona (Berger, 1984), and can accumulate there as twisted magnetic flux emerges from the interior (Pevtsov, Canfield, and McClymont, 1997). It is plausible that helicity accumulation in the corona plays a key role in triggering CMEs (Low, 2001).

Velocities inferred from tracking can also be used to estimate the flux of magnetic helicity across the photosphere (e.g., Chae, 2001, Kusano et al., 2002, 
Proxy Poynting Flux in ARs 987-989
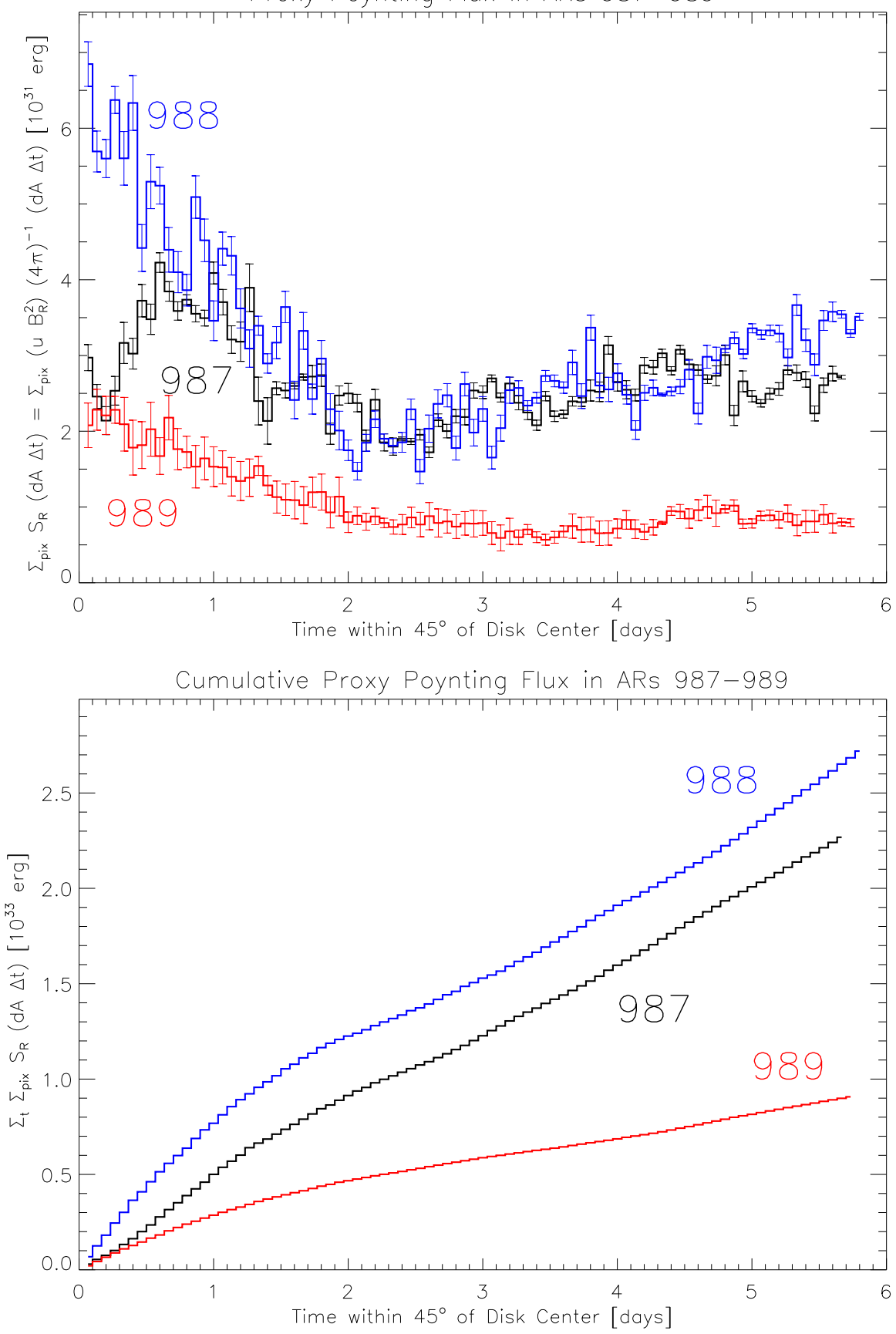

Figure 8. The proxy Poynting flux $\left[S_{R}\right]$ multiplied by pixel area $[\Delta A]$ and the time between magnetograms $[\Delta t]$ (top) and its sum over time (bottom) are both plotted versus time for the three WHI ARs, as each crossed the central solar disk. Error bars for the cumulative plot would be about as large as vertical step sizes. Large values of $\sum_{\text {pix }} S_{R}$ have been associated with flare activity. The sum over $S_{R}$ can provide an order-of-magnitude estimate of the magnetic-energy transport rates. 
Pariat, Demoulin, and Berger , 2005). Using the FLCT flows we estimated for each WHI AR, we computed the helicity flux in that AR over the time interval between each tracked magnetogram pair, separately using $i$ ) Equation (9) with a Green's function method to calculated $\mathbf{A}^{P}$, and ii) the method presented by Pariat, Démoulin, and Berger (2005). Means, medians, and linear fits between the two methods agreed to within 3\% for AR 10987, 1\% for AR 10988, and $6 \%$ for AR 10989. In the top panel of Figure 9, we show the differential fluxes of helicity for the WHI ARs computed from Equation (9), with uncertainties again computed from a running-boxcar calculation of the standard error in flux estimates. (As above, formally propagated errors were too small to appear on these plots.) In the lower panel of Figure 9 we plot the cumulative helicity fluxes of each WHI AR; only every third error bar is plotted, for clarity.

A characteristic magnitude for helicity is the flux in the system squared. In Figure 10, we plot the cumulative helicity fluxes of each WHI AR, normalized by the square of the mean magnetic flux in each AR over the time period it was observed. The unnormalized, cumulative, helicity fluxes for ARs 10988 and 10989 are similar; but since the relative magnetic fluxes of the ARs 10987, 10988, and 10989 are 1.4:1.7:1, normalizing by their squared fluxes implies the characteristic helicity for AR 10989 is substantially higher than for 10988 .

We have reviewed several magnetic parameters of the WHI ARs that might be correlated with flare and CME activity. Characteristic helicity is the only such parameter for which AR 10989 has a larger value than the other ARs. The characteristic helicity fluxes that we found, however, are far below unity, implying that the helicity fluxes did not inject large amounts of twist into the WHI ARs, relative to their magnetic size.

The total helicity present in an AR is the combination of that present in the AR when it emerged and the helicity injected after emergence. By extrapolating non-linear force-free fields (NLFFFs) from a vector magnetogram, the instantaneous helicity content of an AR can be estimated. Petri,Canou, and Amari (2011) have done this for the WHI ARs with SOLIS (Henney et al., 2009) data, and report characteristic helicities (see their Table 1) of $0.0055,0.0041$, and 0.0017 for ARs 10987, 10988, 10989, respectively. Note that all of their characteristic helicity values are on the same order as those we report, but that all of their values are positive; and that their value of the characteristic helicity in AR 10989 is less than those of the other WHI ARs, undermining the hypothesis that characteristic helicity might explain variations in flare and CME productivity.

As an aside, we note that some of our estimates of helicity changes also differ those of Petri,Canou, and Amari (2011) (see the right column of their Figure 23). They show AR 10987 with initially positive helicity that tends to decrease with time, which only partially agrees with our results, which show an initially negative helicity flux followed by a larger, positive helicity flux. Our estimates show negative helicity steadily being added to AR 10988 over time, but Petri,Canou, and Amari (2011) found increasingly positive helicity over one time interval. Finally, the estimates of helicity changes in AR 10989 by Petri,Canou, and Amari (2011) are relatively weak, and of mixed sign; but our results show a steady flux of negative helicity. 

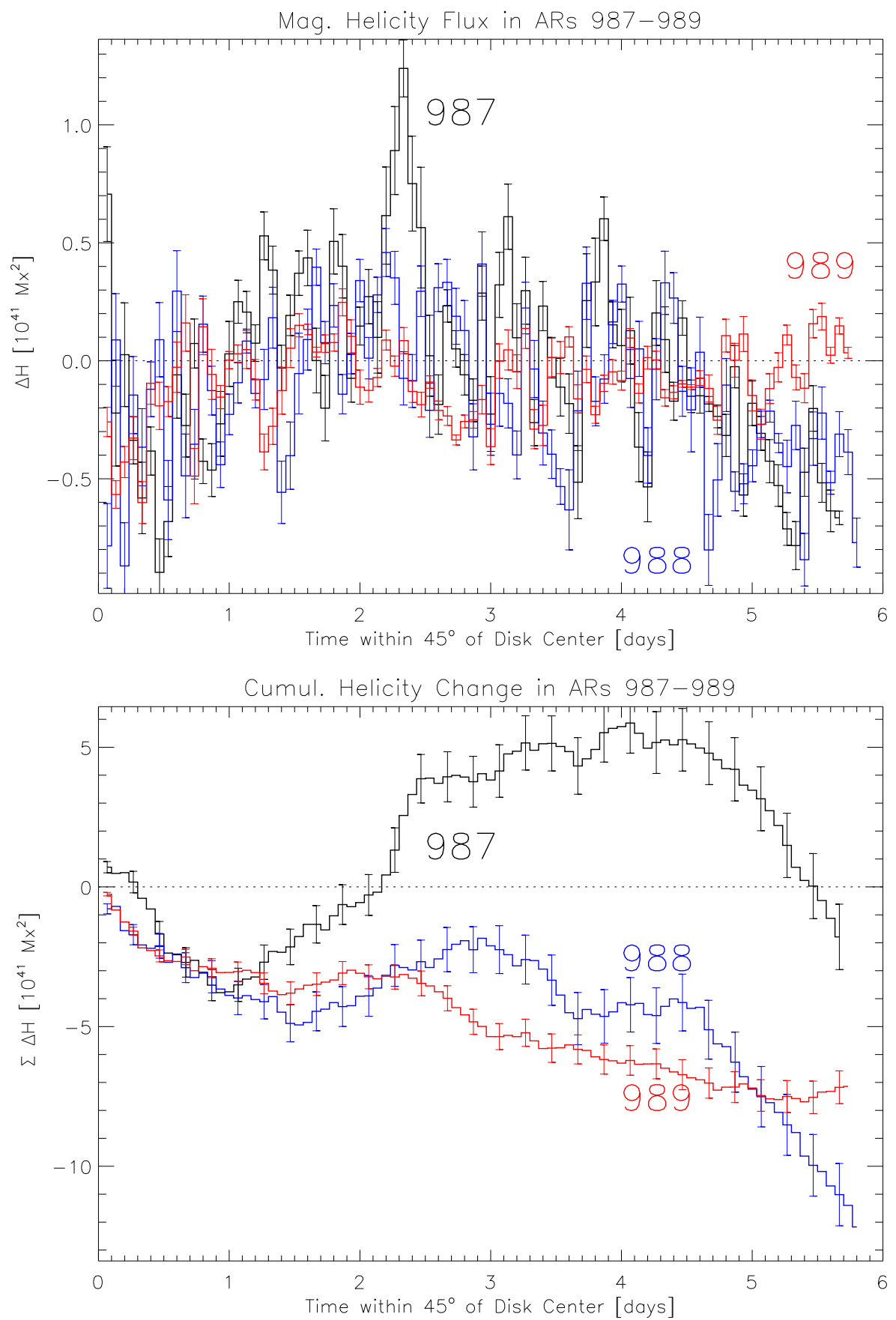

Figure 9. Top: the differential fluxes of helicity for the WHI ARs, with uncertainties. Bottom: The cumulative helicity fluxes of each WHI AR; only every third error bar is plotted, for clarity. 


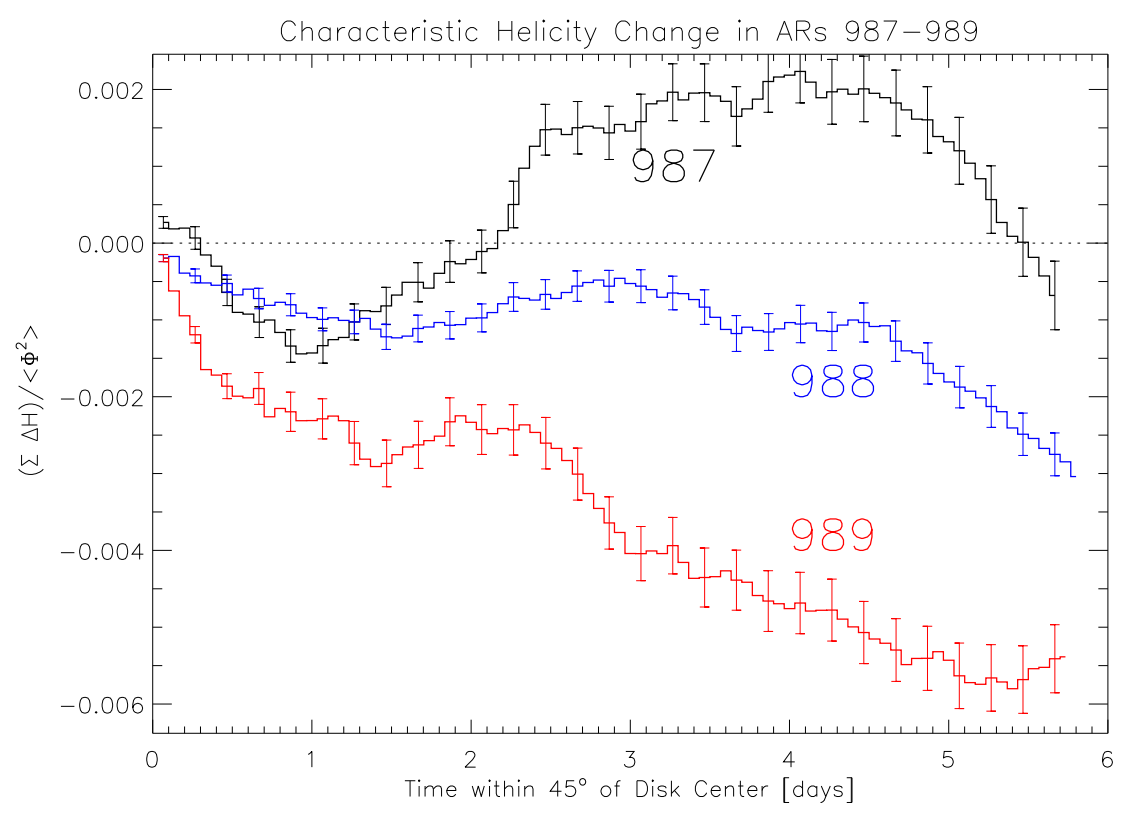

Figure 10. The cumulative characteristic helicity fluxes of each WHI AR: the cumulative helicity flux, normalized by the square of the mean magnetic flux in each AR over the time period it was observed. The unnormalized, cumulative, helicity fluxes for ARs 10988 and 10989 are similar, but the characteristic helicity for AR 10989 is substantially higher than for 10988 .

\subsection{Inductive Flow Estimation}

Flows can be estimated from the induction equation alone, by first applying a Helmholtz decomposition to the horizontal electric field,

$$
c \mathbf{E}_{h}=-\nabla_{h} \phi+\nabla_{h} \times \chi \hat{\mathbf{r}},
$$

and using this result in Equation (1), resulting in a Poisson's equation for $\chi$,

$$
\partial_{t} B_{r}=\nabla_{h}^{2} \chi .
$$

If the electric field is assumed to be ideal, then $c \mathbf{E}_{h}=-\mathbf{u} \times B_{r} \hat{\mathbf{r}}$, and a "Poisson flow" can be derived from $\chi$,

$$
\mathbf{u} B_{r}=-\nabla_{h} \chi
$$

We solved the finite-difference approximation to Equation (12) for the WHI AR magnetogram sequences using Fast Fourier Transforms (FFTs), and then derived Poisson flows. Mean and median Poisson flow speeds for the pair of magnetograms shown in Figure 7 were 91 and $63 \mathrm{~m} \mathrm{sec}^{-1}$, respectively, slow enough to be consistent with the finite-difference approximation.

One problem with this approach is that fluctuations in individual pixels due purely to noise or observational artifacts are ascribed to actual electric fields or 
flows. In contrast, tracking methods such as FLCT and DAVE estimate flows at a given pixel using data from the neighborhood around that pixel (defined with windowing functions), and are therefore less susceptible to spurious fluctuations in field strengths in any individual pixel.

Attempts to ameliorate this shortcoming by smoothing the field difference $\left[\Delta B_{R}\right]$ rapidly degraded the consistency of the Poisson solution with the actual magnetic evolution. This consistency is checked using Equation (7) to calculate the expected magnetic evolution assuming $\mathbf{u} B_{R}$ is known (e.g. Welsch et al., 2007). Temporal averaging of higher-cadence data, however, should counteract the effects of spurious, pixel-scale magnetic fluctuations, but still enable consistency between derived flows and Equation (7).

Unfortunately, statistical agreement between FLCT and Poisson-flow components is poor, with both linear and rank-order correlations below 0.1 for the magnetogram pair in Figure 7, both when weighted by $B_{R}$ and not. Proxy Poynting fluxes derived from tracking and Poisson flow estimates are significantly correlated, but this could arise solely from spatial correlation of $B_{R}^{2}$. Helicity fluxes derived from tracking and Poisson flow estimates are also poorly correlated. Unlike the proxy Poynting flux, helicity-flux density depends sensitively upon direction of flow, since $\mathbf{u}$ is dotted with $\mathbf{A}^{P}$. Given that FLCT and Poissonflow components are weakly correlated, such disagreement in helicity fluxes is not surprising.

Given these disagreements between results from each method, which flow estimate is more credible? Welsch et al. (2009) found flow estimates from the FLCT and DAVE methods - both of which are optical flow techniques, but derive flow estimates in very different ways (Schuck, 2006; Fisher and Welsch, 2008) were significantly correlated. If weighted by $B_{R}$, linear correlation coefficients were $>0.7$ for each flow component for more than 2000 magnetogram pairs in their dataset. In addition, Welsch et al. (2009) found that flow components derived from either FLCT or DAVE persisted in time, with a decorrelation time of approximately six hours (see their Figures 9 and 10). In contrast, Poisson flows for AR 10987 have much lower frame-to-frame correlations than FLCT flows; they essentially decorrelate after one 96-minute interval. Both correlations between flows determined using the independent FLCT and DAVE methods and the persistence of FLCT and DAVE flows in time suggest that the FLCT results found here are more credible than the Poisson flows.

Welsch et al. (2007) tested several velocity-estimation methods using synthetic magnetogram sequences extracted from MHD simulations, in which the flows were known. Later, Welsch and Fisher (2008) tested Poisson-flow estimates with the same synthetic data, and found them to be about as accurate as optical-flow estimates. Since the tracking and Poisson flows were both significantly correlated with the known MHD flows, they were also significantly correlated with each other. The lack of agreement between FLCT and Poisson flows found here for 96minute cadence, full-disk MDI magnetogram data suggests the Poisson method of estimating flows might fail when applied to such data. The Poisson technique might, however, fare better when applied to data with higher cadence and/or higher spatial resolution, such as data from either MDI in its high-resolution mode or HMI. Further study of Poisson flow estimates is planned. 


\section{Discussion}

By all of the statistical predictors of flare activity based upon magnetogram structure and evolution that we investigated, AR 10989 should have been significantly less active than both AR 10987 and 10988. During the intervals that we tracked each AR, however, flare activity in AR 10989 was comparable to activity in the other ARs. Further, Webb et al. (2011) found AR 10989 to be much more CME productive than the other two ARs.

Why did these statistical predictors fail in the case of the WHI ARs? Given that we only consider three ARs here, and that these predictors are purely statistical, we should not be too surprised that there are exceptions. Also, flares are rare events, and the time interval of our observations was limited, so drawing conclusions about activity levels from the small number of flares observed is problematic. While we only analyzed the ARs from the WHI for this study, stronger conclusions regarding relationships (or lack thereof) between ARs' photospheric magnetic properties and flares could presumably be made by studying a larger AR sample. Nonetheless, it would be useful if some physical basis for the exceptions we found could be identified.

Unfortunately, the observations available to us are of limited utility in this regard. First, a practical concern: as noted above, we have only LOS magnetogram sequences for the WHI ARs. Long-duration, regular-cadence vector magnetogram sequences (such as those available from HMI, or that might be available from a network of terrestrial, SOLIS-like instruments) can reveal much more about the structure and evolution of AR magnetic fields.

Second, another practical concern: we are largely ignorant of the WHI active regions' magnetic structure and coronal activity over much of their lives. A significant component of AR 10989's activity occurred both before and after we tracked it across the central solar disk. Because the structure and evolution of its photospheric magnetic field could only be reliably estimated while on the central disk, we can only speculate about this field away from disk center. Welsch et al. (2009) showed that AR magnetic fields can evolve substantially over the course of 24 hours, and Longcope et al. (2005) and Schrijver et al. (2005) estimated coronal energy storage times on the order of a day. If the field of AR 10989 evolved significantly between its time on the central disk and the pre- and posttracking intervals, its photospheric magnetic structure during those more active invtervals might have been more consistent with statistical expectations based upon its coronal activity level. Similarly, we often only have hints about flares and CMEs produced by ARs beyond the limb. For instance, STEREO-A data show that on 05 April, AR 10987 at longitude W105 produced a CME just before 16:00UT with a speed estimated from LASCO data at $962 \mathrm{~km} \mathrm{~s}^{-1}$, associated with an A-class GOES flare. (See Nitta's excellent compilation of data for this and other events at http://www.Imsal.com/nitta/movies/flares_euvi/) Burkepile et al. (2004) has shown that GOES flux is correlated with CME kinetic energy, with a linear correlation coefficient of 0.74. Similarly, the events analyzed by Qiu and Yurchyshyn (2005) show that GOES flux is correlated with CME speed: we find linear and rank-order correlation coefficients of 0.79 and 0.54 , respectively, in their sample of 13 events. These results suggest that the A-class flare observed 
by GOES was most likely a partially occulted strong C-class or weak M-class flare. To address this ignorance, having more extensive magnetograph, X-ray, in-situ, and EUV coverage of the Sun would be useful. A satellite in co-rotation with the Sun could provide comprehensive observations of ARs from birth to death; and a constellation of satellites might provide full, " $4 \pi$ " coverage.

Third, studying photospheric data alone neglects aspects of coronal magnetic structure that are relevant to flaring. For instance, the large-scale structure of the coronal magnetic field, including perhaps the proximity of an AR to open magnetic fields, might play a key role in CME productivity. Sterling and Moore (2001) argued that open flux near NOAA AR 8210 enabled repeated, homologous ejections (see their Figure 3). Similarly, Liu (2007) reported that CMEs underneath the streamer belt tended to be substantially slower than CMEs outside the belt, suggesting that the global magnetic environment around ARs can play a significant role in the CME process. Global-scale potential-field source-surface (PFSS) modeling by Petri,Canou, and Amari (2011) demonstrates that AR 10989 lies almost completely outside the streamer belt (see their Fig. 21), perhaps easing the ejection of coronal fields into the heliosphere as CMEs. Differences in large-scale coronal magnetic environment might partially explain why flare activity levels were broadly similar among the WHI ARs, but CME activity levels were not. (It is also true that flares and CMEs appear to be distinct phenomena: very large flares are almost always associated with CMEs, but that the association is weaker with weaker flares (Andrews, 2003).) It would be useful to study objective, quantitative measures of "proximity" between open flux and ARs, and correlations between CME activity and such proximity measures. Hudson and $\mathrm{Li}(2010)$ noted that the flare-to-CME ratio increased this solar minimum relative to the last, implying the corona "was relatively easy to disrupt" during this minimum, also suggesting global properties of the coronal magnetic field might play some role in the CME process.

Fourth, properties of photospheric magnetic fields might be essentially unrelated to properties of the coronal magnetic field that actually generate flares and CMEs. Having analyzed a large sample of vector magnetograms, Leka and Barnes (2007) suggested "the state of the photospheric magnetic field at any given time has limited bearing on whether that region will be flare productive." (Based upon the case studies here, we might add the evolution of the line-ofsight photospheric magnetic field over any given time interval also has limited bearing on whether that region will be flare productive - but we have not analyzed enough cases to justify such a conclusion.) Efforts to measure properties of coronal magnetic fields using IR (SOLARC: Lin, Kuhn, and Coulter, 2004; CoMP: Tomczyk and McIntosh, 2009; ATST: Cargill, 2009) and radio (FASR: Cargill, 2009) instruments could lead to breakthroughs in our understanding of flare and CME physics.

Finally, leaving aside all observational limitations, flares might be unpredictable for more fundamental reasons, a possibility also noted by Schrijver (2009).

Acknowledgements The authors owe thanks to many people: the WHI Team for inviting BTW to participate in the Second WHI Workshop; the organizers of this Topical Issue of Solar 
Physics; the SOHO/MDI and RHESSI teams for making their databases available and easy to use; and the American taxpayers, for their financial support of this work. MDI is funded through NASAs Solar and Heliospheric Physics program; SOHO is a project of international cooperation between ESA and NASA. This research has made use of NASA's Astrophysics Data System Service. BTW acknowledges support from NSF awards ATM-0752597 and AGS1024862. JMM acknowledges support from NASA grants NAS5-98033, NNX08AJ18G and NNX08AI56G.

\section{References}

Abramenko, V.I.: 2005, Relationship between Magnetic Power Spectrum and Flare Productivity in Solar Active Regions. Astrophys. J. 629, 1141-1149. doi:10.1086/431732.

Andrews, M.D.: 2003, A Search for CMEs Associated with Big Flares. Solar Phys. 218, 261 279. doi:10.1023/B:SOLA.0000013039.69550.bf.

Barnes, G., Leka, K.D.: 2008, Evaluating the Performance of Solar Flare Forecasting Methods. Astrophys. J. Lett. 688, L107-L110. doi:10.1086/595550.

Berger, M.A.: 1984, Rigorous new limits on magnetic helicity dissipation in the solar corona. Geophys. Astrophys. Fluid Dynamics 30, 79-104.

Berger, M.A., Field, G.B.: 1984, The topological properties of magnetic helicity. J. Fl. Mech. 147, $133-148$

Burkepile, J.T., Hundhausen, A.J., Stanger, A.L., St. Cyr, O.C., Seiden, J.A.: 2004, Role of projection effects on solar coronal mass ejection properties: 1. A study of CMEs associated with limb activity. J. Geophys. Res. (Space Phys.) 109(PA18), 3103. doi:10.1029/2003JA010149.

Cargill, P.J.: 2009, Coronal Magnetism: Difficulties and Prospects. Space Sci. Rev. 144, 413421. doi:10.1007/s11214-008-9446-9.

Chae, J.: 2001, Observational determination of the rate of magnetic helicity transport through the solar surface via the horizontal motion of field line footpoints. Astrophys. J. Lett. 560, L95-L98.

Christe, S., Krucker, S., Lin, R.P.: 2008a, Hard X-Rays Associated with Type III Radio Bursts. Astrophys. J. Lett. 680, L149-L152. doi:10.1086/589971.

Christe, S., Hannah, I.G., Krucker, S., McTiernan, J., Lin, R.P.: 2008b, RHESSI Microflare Statistics. I. Flare-Finding and Frequency Distributions. Astrophys. J. 677, 1385-1394. doi:10.1086/529011.

Démoulin, P., Berger, M.A.: 2003, Magnetic Energy and Helicity Fluxes at the Photospheric Level. Solar Phys. 215, 203-215.

DeForest, C.E., Hagenaar, H.J., Lamb, D.A., Parnell, C.E., Welsch, B.T.: 2007, Solar Magnetic Tracking. I. Software Comparison and Recommended Practices. Astrophys. J. 666, $576-$ 587. doi:10.1086/518994.

Falconer, D.A., Moore, R.L., Gary, G.A.: 2003, A measure from line-of-sight magnetograms for prediction of coronal mass ejections. J. Geophys. Res. (Space Phys.) 108(A10), 11-1. doi:10.1029/2003JA010030.

Falconer, D.A., Moore, R.L., Gary, G.A.: 2006, Magnetic Causes of Solar Coronal Mass Ejections: Dominance of the Free Magnetic Energy over the Magnetic Twist Alone. Astrophys. J. 644, $1258-1272$. doi: $10.1086 / 503699$.

Fisher, G.H., Welsch, B.T.: 2008, FLCT: A Fast, Efficient Method for Performing Local Correlation Tracking. In: Subsurface and Atmospheric Influences on Solar Activity, Howe, R. Komm, R.W., Balasubramaniam, K.S., Petrie, G.J.D. (eds.) Astron. Soc. Pacific CS, San Francisco. 383, 373-380; also arXiv:07124289.

Forbes, T.G.: 2000, A review on the genesis of coronal mass ejections. J. Geophys. Res. 105, $23153-23166$

Gosling, J.T.: 1993, The solar flare myth. JGR 98(A11), 18937-18949.

Hagenaar, H.J., Schrijver, C.J., Title, A.M.: 1997, The Distribution of Cell Sizes of the Solar Chromospheric Network. Astrophys. J. 481, 988. doi:10.1086/304066.

Hagenaar, H., Schrijver, C., Title, A., Shine, R.: 1999, Dispersal of magnetic flux in the quiet solar photosphere. ApJ 511, $932-944$. 
Henney, C.J., Keller, C.U., Harvey, J.W., Georgoulis, M.K., Hadder, N.L., Norton, A.A., Raouaf, N., Toussaint, R.M.: 2009, SOLIS Vector Spectromagnetograph: Status and Science. In: SOLAR POLARIZATION 5: In Honor of Jan Olof Stenflo, S. V. Berdyugina, K. N. Nagendra, R. Ramelli (ed.) Astron. Soc. Pacific CS, San Francisco. 405, 47.

Hudson, H.S., Li, Y.: 2010, Flare and CME Properties and Rates at Sunspot Minimum. In: S. R. Cranmer, J. T. Hoeksema, J. L. Kohl (ed.) SOHO 23: Understanding a Peculiar Solar Minimum, Astron. Soc. Pacific CS, San Francisco. 428, 153.

Kusano, K., Maeshiro, T., Yokoyama, T., Sakurai, T.: 2002, Measurement of magnetic helicity injection and free energy loading into the solar corona. Astrophys. J. 577, 501-512.

Leka, K.D., Barnes, G.: 2007, Photospheric Magnetic Field Properties of Flaring versus Flarequiet Active Regions. IV. A Statistically Significant Sample. Astrophys. J. 656, $1173-$ 1186 .

Li, Y., Lynch, B.J., Welsch, B.T., Stenborg, G.A., Luhmann, J.G., Fisher, G.H., Liu, Y., Nightingale, R.W.: 2010, Sequential Coronal Mass Ejections from AR8038 in May 1997. Solar Phys. 264, 149-164. doi:10.1007/s11207-010-9547-y.

Lin, H., Kuhn, J.R., Coulter, R.: 2004, Coronal Magnetic Field Measurements. Astrophys. J. Lett. 613, L177-L180. doi:10.1086/425217.

Lin, R.P., Dennis, B.R., Hurford, G.J., Smith, D.M., Zehnder, A., Harvey, P.R., Curtis, D.W., Pankow, D., Turin, P., Bester, M., Csillaghy, A., Lewis, M., Madden, N., van Beek, H.F., Appleby, M., Raudorf, T., McTiernan, J., Ramaty, R., Schmahl, E., Schwartz, R., Krucker, S., Abiad, R., Quinn, T., Berg, P., Hashii, M., Sterling, R., Jackson, R. Pratt, R., Campbell, R.D., Malone, D., Landis, D., Barrington-Leigh, C.P., Slassi-Sennou, S., Cork, C., Clark, D., Amato, D., Orwig, L., Boyle, R., Banks, I.S., Shirey, K., Tolbert, A.K., Zarro, D., Snow, F., Thomsen, K., Henneck, R., McHedlishvili, A., Ming, P., Fivian, M., Jordan, J., Wanner, R., Crubb, J., Preble, J., Matranga, M., Benz, A., Hudson, H., Canfield, R.C., Holman, G.D., Crannell, C., Kosugi, T., Emslie, A.G., Vilmer, N., Brown, J.C., Johns-Krull, C., Aschwanden, M., Metcalf, T., Conway, A.: 2002, The Reuven Ramaty High-Energy Solar Spectroscopic Imager (RHESSI). Solar Phys. 210, 3-32. doi:10.1023/A:1022428818870.

Linker, J.A., Mikić, Z., Lionello, R., Riley, P., Amari, T., Odstrcil, D.: 2003, Flux cancellation and coronal mass ejections. Physics of Plasmas 10, 1971-1978. doi:10.1063/1.1563668.

Liu, Y.: 2007, Halo Coronal Mass Ejections and Configuration of the Ambient Magnetic Fields. Astrophys. J. 654, L171-L174. doi:10.1086/511385.

Liu, Y., Norton, A.A., Scherrer, P.H.: 2007, A Note on Saturation Seen in the MDI/SOHO Magnetograms. Solar Phys. 241, 185-193. doi:10.1007/s11207-007-0296-5.

Livi, S.H.B., Wang, J., Martin, S.F.: 1985, The cancellation of magnetic flux. I - On the quiet sun. Austral. J. Phys. 38, 855-873.

Longcope, D.W., McKenzie, D., Cirtain, J., Scott, J.: 2005, Observations of separator reconnection to an emerging active region. Astrophys. J. 630, 596-614.

Low, B.C.: 2001, Coronal mass ejections, magnetic flux ropes, and solar magnetism. J. Geophys. Res. 106, 25141-25164. doi:10.1029/2000JA004015.

Martin, S.F.: 1998, Conditions for the Formation and Maintenance of Filaments - (Invited Review). Solar Phys. 182, $107-137$.

Pariat, E., Demoulin, P., Berger, M.A.: 2005, Photospheric flux density of magnetic helicity. Astron. Astrophys. 439, 1191-1203. doi:10.1051/0004-6361:20052663.

Parnell, C.E., Deforest, C.E., Hagenaar, H.J., Johnston, B.A., Lamb, D.A., Welsch, B.T.: 2009, A Power-law Distribution of Solar Magnetic Fields Over More Than Five Decades in Flux. Astrophys. J. 698, 75-82.

Petrie, G.J.D., Canou, A., Amari, T.: 2011, Nonlinear Force-Free and Potential-Field Models of Active-Region and Global Coronal Fields during the Whole Heliosphere Interval. Solar Phys. Online First, - . doi:10.1007/s11207-010-9687-0.

Pevtsov, A.A., Canfield, R.C., McClymont, A.N.: 1997, On the subphotospheric origin of coronal electric currents. Astrophys. J. 481, 973-977.

Qiu, J., Yurchyshyn, V.B.: 2005, Magnetic Reconnection Flux and Coronal Mass Ejection Velocity. Astrophys. J. Lett. 634, L121-L124. doi:10.1086/498716.

Scherrer, P., Bogart, R.S., Bush, R.I., Hoeksema, J.T., Kosovichev, A., Schou, J., Rosenberg, W., Springer, L., Tarbell, T., Title, A., Wolfson, C., Zayer, I., The MDI Engineering Team: 1995, The solar oscillations investigation - michelson doppler imager. Solar Phys. 162 $129-188$. 
Schrijver, C.J.: 2007, A Characteristic Magnetic Field Pattern Associated with All Major Solar Flares and Its Use in Flare Forecasting. Astrophys. J. Lett. 655, L117-L120. doi:10.1086/511857.

Schrijver, C.J.: 2009, Driving major solar flares and eruptions: A review. Adv. Space Res. 43, 739 - 755. doi:10.1016/j.asr.2008.11.004.

Schrijver, C.J., DeRosa, M.L., Title, A.M., Metcalf, T.R.: 2005, The Nonpotentiality of ActiveRegion Coronae and the Dynamics of the Photospheric Magnetic Field. Astrophys. J. 628, $501-513$.

Schuck, P.W.: 2006, Tracking Magnetic Footpoints with the Magnetic Induction Equation. Astrophys. J. 646, 1358-1391. doi:10.1086/505015.

Schuck, P.W.: 2008, Tracking Vector Magnetograms with the Magnetic Induction Equation. Astrophys. J. 683, 1134-1152. doi:10.1086/589434.

Sterling, A.C., Moore, R.L.: 2001, Internal and external reconnection in a series of homologous solar flares. J. Geophys. Res. 106, 25227-25238. doi:10.1029/2000JA004001.

Tomczyk, S., McIntosh, S.W.: 2009, Time-Distance Seismology of the Solar Corona with CoMP. Astrophys. J. 697, 1384-1391. doi:10.1088/0004-637X/697/2/1384.

Wang, D., Zhang, M., Li, H., Zhang, H.Q.: 2009, A Cross-Comparison of Cotemporal Magnetograms Obtained with MDI/SOHO and SP/ Hinode. Solar Phys. 260, 233-244. doi:10.1007/s11207-009-9441-7.

Webb, D.F., Cremades, H., Sterling, A.C, Mandrini, C.H., Dasso, S., Gibson, S.E., Haber, D.A., Komm, R.W., Petrie, G.J.D., McIntosh, P.S., Welsch, B.T., Simon, S.P.: 20011, The Global Context of Solar Activity During the Whole Heliosphere Interval Campaign. Solar Phys. (in preparation), - .

Welsch, B.T.: 2006, Magnetic flux cancellation and coronal magnetic energy. Astrophys. J. 638, 1101

Welsch, B.T., Fisher, G.H.: 2008, Surface Flows From Magnetograms. In: Subsurface and Atmospheric Influences on Solar Activity, Howe, R., Komm, R.W., Balasubramaniam, K.S., Petrie, G.J.D. (eds.) Astron. Soc. Pacific CS, San Francisco. 383, $19-30 ;$ also arXiv:07100546.

Welsch, B.T., Li, Y.: 2008, On the Origin of Strong-Field Polarity Inversion Lines. In: Subsurface and Atmospheric Influences on Solar Activity, Howe, R., Komm, R.W., Balasubramaniam, K.S., Petrie, G.J.D. (eds.) Astron. Soc. Pacific CS, San Francisco. 383, 429-437; also arXiv:07100562.

Welsch, B.T., Longcope, D.W.: 2003, Magnetic helicity injection by horizontal flows in the quiet sun: I. mutual helicity flux. Astrophys. J. $\mathbf{5 8 8 .}$

Welsch, B.T., Fisher, G.H., Abbett, W.P., Régnier, S.: 2004, ILCT: Recovering photospheric velocities from magnetograms by combining the induction equation with local correlation tracking. Astrophys. J. 610, 1148.

Welsch, B.T., Abbett, W.P., DeRosa, M.L., Fisher, G.H., Georgoulis, K. M. K. Kusano, Longcope, D.W., Ravindra, B., Schuck, P.W.: 2007, Tests and comparisons of velocity inversion techniques. Astrophys. J. 670, 1434-1452.

Welsch, B.T., Li, Y., Schuck, P.W., Fisher, G.H.: 2009, What is the Relationship Between Photospheric Flow Fields and Solar Flares? Astrophys. J. 705, 821-843. doi:10.1088/0004-637X/705/1/821. 
SOLA: ms_rev2.tex; 4 June 2018; 12:43; p. 30 


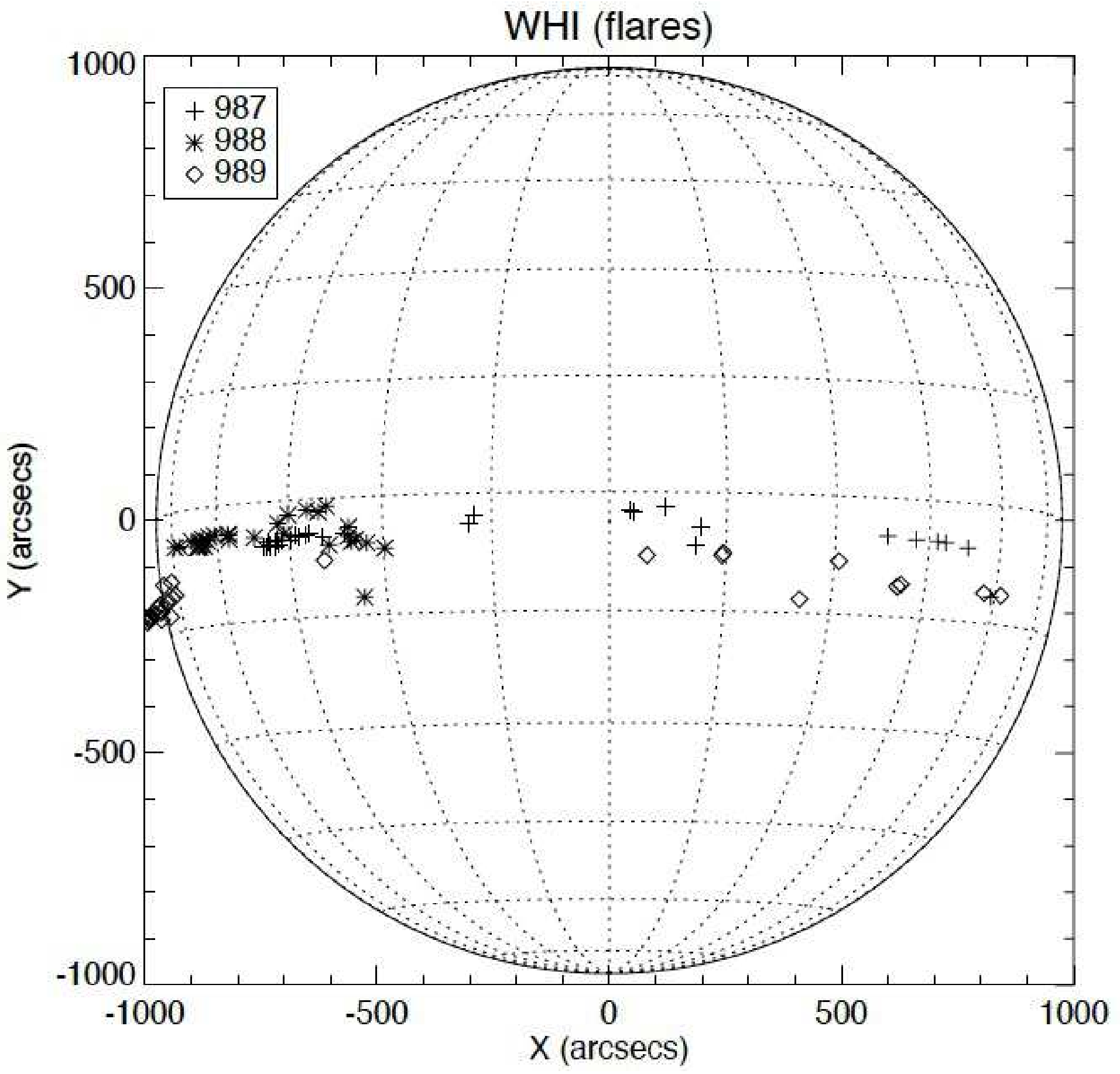

